Publications of the Astronomical Society of the Pacific, 119: 855-870, 2007 August

(C) 2007. The Astronomical Society of the Pacific. All rights reserved. Printed in U.S.A.

\title{
The James Clerk Maxwell Telescope Legacy Survey of Nearby Star-forming Regions in the Gould Belt
}

\author{
D. Ward-Thompson, ${ }^{1}$ J. Di Francesco, ${ }^{2}$ J. Hatchell,${ }^{3}$ M. R. Hogerheijde, ${ }^{4}$ D. Nutter,${ }^{1}$ P. Bastien, ${ }^{5}$ S. Basu, ${ }^{6}$ \\ I. Bonnell ${ }^{7}$ J. Bowey, ${ }^{8}$ C. Brunt ${ }^{3}$ J. Buckle, ${ }^{9}$ H. Butner,${ }^{10}$ B. Cavanagh, ${ }^{11}$ A. Chrysostomou, ${ }^{11,12}$ E. Curtis, ${ }^{9}$ \\ C. J. Davis, ${ }^{11}$ W. R. F. Dent,${ }^{13}$ E. van Dishoeck, ${ }^{4}$ M. G. Edmunds, ${ }^{1}$ M. Fich, ${ }^{14}$ J. Fiege, ${ }^{15}$ L. Fissel, ${ }^{16}$ P. Friberg, ${ }^{11}$ \\ R. Friesen, ${ }^{2,17}$ W. Frieswijk, ${ }^{18}$ G. A. Fuller, ${ }^{19}$ A. Gosling,${ }^{20}$ S. Graves, ${ }^{9}$ J. S. Greaves, ${ }^{7}$ F. Helmich, ${ }^{18}$ R. E. Hills, ${ }^{9}$
} W. S. Holland, ${ }^{13}$ M. Houde, ${ }^{6}$ R. Jayawardhana, ${ }^{16}$ D. Johnstone, ${ }^{2,17}$ G. Joncas, ${ }^{21}$ H. KirK, ${ }^{2,17}$ J. M. Kirk, ${ }^{1}$ L. B. G. Knee, ${ }^{2}$ B. Matthews, ${ }^{2}$ H. Matthews, ${ }^{22}$ C. Matzner, ${ }^{16}$ G. H. Moriarty-Schieven, ${ }^{2,11}$ D. Naylor,${ }^{23}$ R. Padman, ${ }^{9}$ R. Plume, ${ }^{24}$ J. M. C. Rawlings, ${ }^{8}$ R. O. Redman, ${ }^{2}$ M. Reid, ${ }^{25}$ J. S. Richer, ${ }^{9}$ R. Shipman, ${ }^{4}$ R. J. Simpson, ${ }^{1}$ M. SpaAns, ${ }^{4}$ D. Stamatellos, ${ }^{1}$ Y. G. Tsamis, ${ }^{8}$ S. Viti, ${ }^{8}$ B. Weferling, ${ }^{11}$ G. J. White, ${ }^{26,27}$ A. P. Whitworth, ${ }^{1}$ J. Wouterloot, ${ }^{11}$ J. Yates, ${ }^{8}$ And M. Zhu ${ }^{2,11}$

Received 2007 May 24; accepted 2007 June 29; published 2007 September 5

\begin{abstract}
This paper describes a James Clerk Maxwell Telescope (JCMT) legacy survey that has been awarded roughly $500 \mathrm{hr}$ of observing time to be carried out from 2007 to 2009. In this survey, we will map with SCUBA2 (Submillimetre Common-User Bolometer Array 2) almost all of the well-known low-mass and intermediate-mass star-forming regions within $0.5 \mathrm{kpc}$ that are accessible from the JCMT. Most of these locations are associated with the Gould Belt. From these observations, we will produce a flux-limited snapshot of star formation near the Sun, providing a legacy of images, as well as point-source and extended-source catalogs, over almost $700 \mathrm{deg}^{2}$ of sky. The resulting images will yield the first catalog of prestellar and protostellar sources selected by submillimeter continuum emission, and should increase the number of known sources by more than an order of magnitude. We will also obtain with the array receiver HARP (Heterodyne Array Receiver Program) CO maps, in three CO isotopologues, of a large typical sample of prestellar and protostellar sources. We will then map the brightest hundred sources with the SCUBA-2 polarimeter (POL-2), producing the first statistically significant set of polarization maps in the submillimeter. The images and source catalogs will be a powerful reference set for astronomers, providing a detailed legacy archive for future telescopes, including ALMA, Herschel, and JWST.
\end{abstract}

\section{INTRODUCTION}

\subsection{Nearby Star Formation}

Understanding star formation is a crucial goal of astronomy. Star formation plays a pivotal role in most aspects of astronomy, from the formation and evolution of galaxies to the origins of extrasolar planets and the potential for life elsewhere in our Galaxy. Our knowledge of the star formation process has increased dramatically due to the advent of sensitive far-infrared

\footnotetext{
${ }^{1}$ School of Physics and Astronomy, Cardiff University, Cardiff, UK.

${ }^{2}$ Herzberg Institute of Astrophysics, National Research Council of Canada, Victoria, BC, Canada.

${ }^{3}$ School of Physics, University of Exeter, Exeter, UK.

${ }^{4}$ Leiden Observatory, Leiden University, Leiden, Netherlands.

${ }^{5}$ Département de Physique et Observatoire due Mont Mégantic, Université de Montréal, Montréal, QC, Canada.

${ }^{6}$ Physics and Astronomy Department, University of Western Ontario, London, ON, Canada.

${ }^{7}$ Scottish Universities Physics Alliance, Physics and Astronomy, University of St. Andrews, North Haugh, St. Andrews, Fife, UK.

${ }^{8}$ Department of Physics and Astronomy, University College London, London, UK.
}

and submillimeter detectors, but has suffered from the piecemeal fashion in which such observations have been undertaken to date. We describe here a project that aims to produce a large and unbiased sample of star-forming molecular material in the solar vicinity at relatively high resolution $\left(8^{\prime \prime}-14^{\prime \prime}\right)$.

To understand star formation, we need to probe the physical conditions of molecular clouds before and during the star for-

\footnotetext{
${ }^{9}$ Cavendish Laboratory, Cambridge University, Cambridge, UK.

${ }^{10}$ Department of Physics and Astronomy, James Madison University, Harrisonburg, VA.

${ }^{11}$ Joint Astronomy Center, University Park, Hilo, Hawaii.

${ }^{12}$ School of Physics, Astronomy and Mathematics, University of Hertfordshire, Hatfield, UK.

${ }^{13}$ UK Astronomy Technology Centre, Royal Observatory, Blackford Hill, Edinburgh, UK.

${ }^{14}$ Department of Physics and Astronomy, University of Waterloo, Waterloo, ON, Canada.

${ }^{15}$ Department of Physics and Astronomy, University of Manitoba, Winnipeg, MB, Canada.

${ }^{16}$ Department of Astronomy and Astrophysics, University of Toronto, Toronto, ON, Canada.
} 


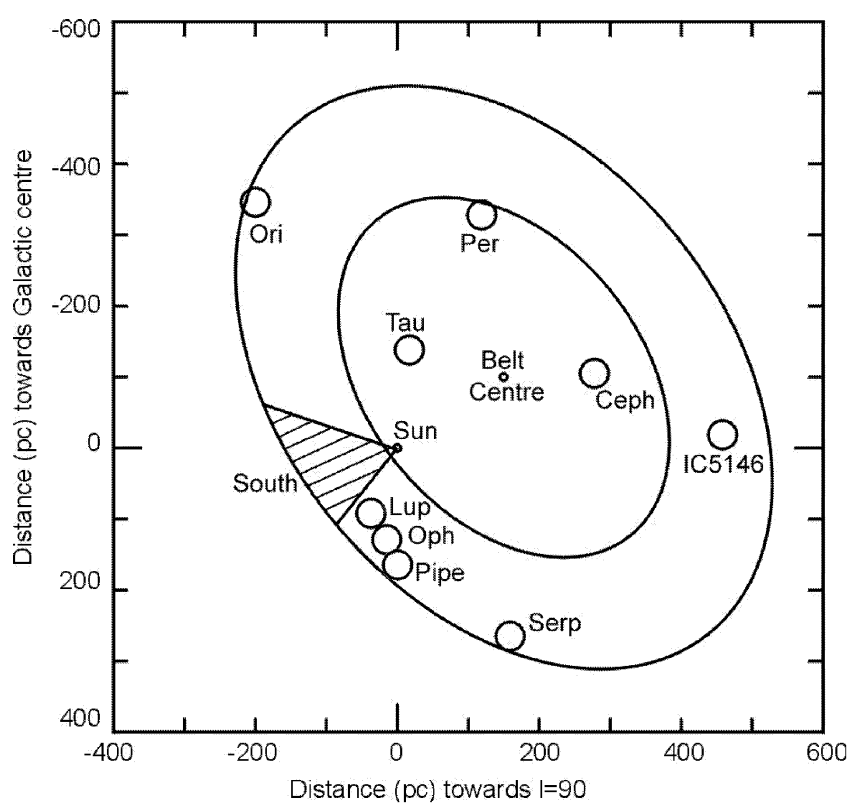

FIG. 1.-Diagram illustrating the Gould Belt and the positions of each of its constituent clouds relative to the Sun. The shaded area represents that part of the Belt not visible from the JCMT.

mation process. Although near-IR images can tell us a great deal about the results of star formation, the objects that are visible are too old to assess the crucial conditions in which star formation originates. We need to illuminate the earliest conditions in order to understand the formation process (e.g., Di Francesco et al. 2007; Ward-Thompson et al. 2007).

Submillimeter continuum imaging selects the very earliest stages of star formation, because it traces the high column densities of dust, even when that dust is at low temperatures, within star-forming cores and allows important physical parameters such as density to be traced in detail. From its earliest days, the James Clerk Maxwell Telescope (JCMT) has been mapping submillimeter continuum emission from star-forming regions (e.g., Ward-Thompson et al. 1989, 1995). The JCMT identified first the youngest known protostars (Class 0 objects; André et al. 1993) and molecular cloud cores on the verge of collapse to form protostars (prestellar cores; Ward-Thompson et al. 1994).

The JCMT also helped determine that the prestellar core mass function mimics the initial mass function (IMF), indi-

\footnotetext{
${ }^{17}$ Department of Physics and Astronomy, University of Victoria, Victoria, BC, Canada.

${ }^{18}$ SRON, Netherlands Institute for Space Research, Groningen, Netherlands.

${ }^{19}$ School of Physics and Astronomy, University of Manchester, Manchester, UK.

${ }^{20}$ Astrophysics Group, Department of Physics, Oxford University, Oxford, UK.

${ }^{21}$ Department de Physique et Observatoire du Mont Megantic, Universite Laval, QC, Canada.
}

cating that it may be determined at the very beginning of the star formation process- this was the first observational breakthrough in understanding its origin in nearly 50 years (Johnstone et al. 2000; Motte et al. 2001; Nutter \& Ward-Thompson 2007). Many large area continuum mapping surveys of starforming regions have also been carried out with the Submillimetre Common-User Bolometer Array (SCUBA) on JCMT (e.g., Johnstone \& Bally 1999; Pierce-Price et al. 2000; Johnstone et al. 2000, 2001, 2006; Hatchell et al. 2005, 2007; Nutter et al. 2005, 2006; Nutter \& Ward-Thompson 2007; J. Kirk et al. 2005; Moriarty-Schieven et al. 2006). Many of these regions lie in a ring around the sky, coincident with the Gould Belt.

\subsection{The Gould Belt}

The Gould Belt is a ring of nearby O-type stars inclined at about $20^{\circ}$ to the Galactic plane. It was in fact first discovered in the Southern Hemisphere by J. Herschel (1847), who noted that many of the brightest stars in the Southern sky lie in a band that is inclined to the plane of the Galaxy. Subsequently, Gould (1879) traced the northern part of the band, thereby completing the ring.

The Gould Belt is centered on a point $\sim 200 \mathrm{pc}$ from the Sun and is about 350 pc in radius (e.g., Clube 1967; Stothers \& Frogel 1974; Comeron et al. 1992; de Zeeuw et al. 1999; Pöppel 2001). Figure 1 shows a schematic of the Gould Belt, and Figure 2 shows its projection onto the plane of the sky, showing the inclination to the Galactic plane.

The formation mechanism of the Belt remains something of a mystery. One hypothesis is that it may be the result of a high-velocity cloud impacting the Galactic plane (Comeron \& Torra 1992, 1994; Guillout et al. 1998). An alternative possibility is a local massive supernova remnant or stellar wind interacting with a large molecular cloud (Blaauw 1991).

Whatever the cause, the Gould Belt is a highly active ring of nearby star formation, and most of the local star-forming molecular clouds are associated with it, including Taurus, Auriga, Orion, Lupus, Ophiuchus, Scorpius, Serpens, and Perseus. Study of the star formation within the Gould Belt sheds light on the process of star formation within these respective clouds. Moreover, it may also help to shed light on the whole of the Gould Belt itself. For example, accurate dating of the bursts of star formation around the Belt may be able to test the various formation mechanisms of the Gould Belt.

\footnotetext{
${ }^{22}$ National Research Council of Canada, Dominion Radio Astrophysical Observatory, Penticton, BC, Canada.

${ }^{23}$ Department of Physics, University of Lethbridge, Lethbridge, AB, Canada.

${ }^{24}$ Department of Physics and Astronomy, University of Calgary, Calgary, AB, Canada.

${ }^{25}$ Department of Physics and Astronomy, McMaster University, Hamilton, ON, Canada.

${ }^{26}$ Department of Physics and Astronomy, Open University, Milton Keynes, UK.

${ }^{27}$ Science and Technology Facilities Council, Rutherford Appleton Laboratory, Chilton, Didcot, UK.
} 


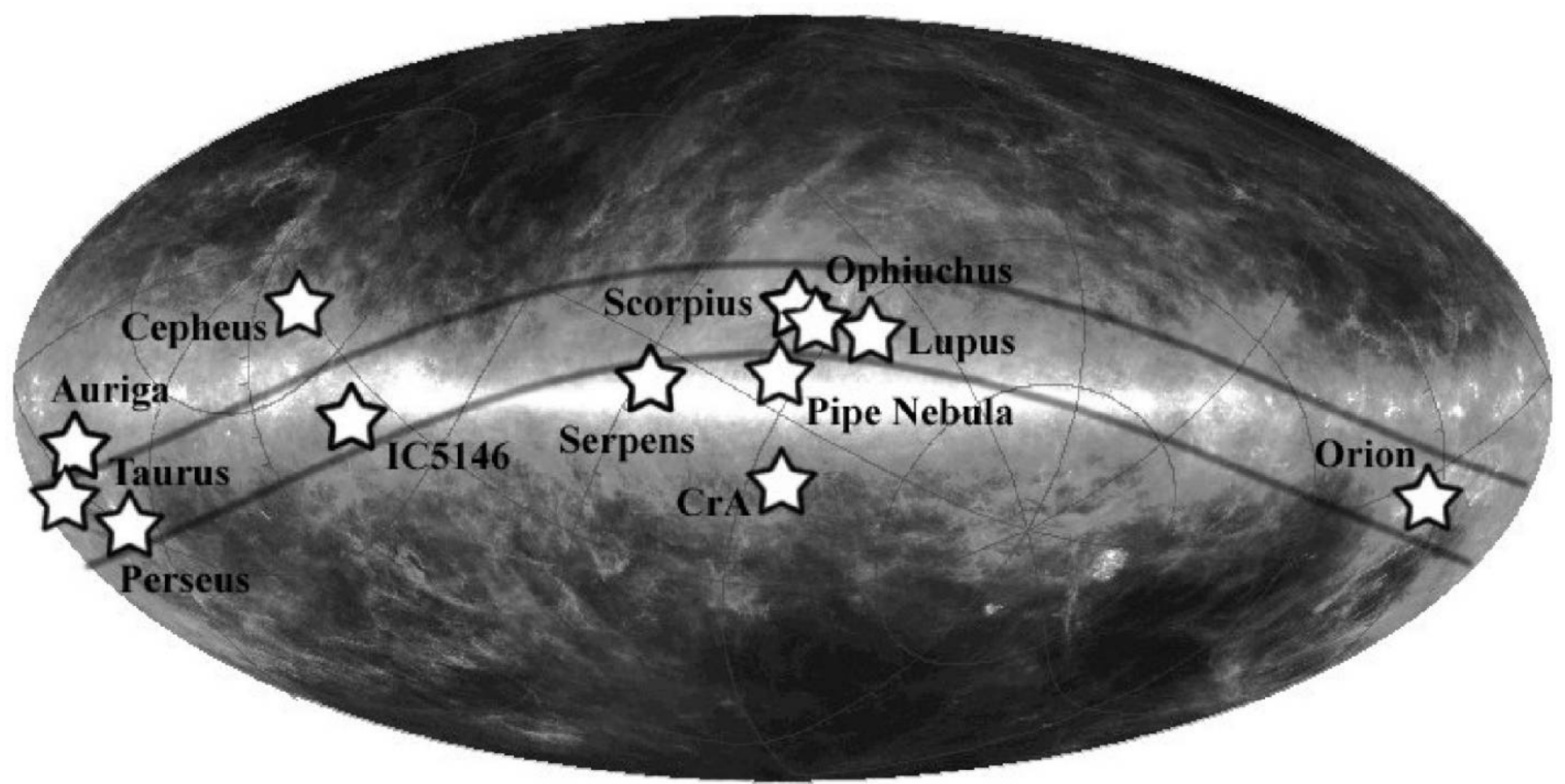

FIG. 2.-All-sky image of IRAS $100 \mu \mathrm{m}$ data plotted in Galactic coordinates, showing the projection of the Gould Belt onto the plane of the sky and the positions of the various clouds.

\subsection{A New Era for JCMT}

The JCMT is currently undergoing a complete overhaul of its instrumentation, including a new bolometer array camera, the Submillimetre Common-User Bolometer Array 2 (SCUBA-2; Holland et al. 2006), with an imaging polarimeter, the SCUBA2 Polarimeter (POL-2; Bastien et al. 2005), and a heterodyne array receiver, the Heterodyne Array Receiver Programme for $B$-band (HARP; Smith et al. 2003). The combination of SCUBA2, HARP, and POL-2 provide a powerful tool set with which to study star formation.

SCUBA-2 is an innovative 10,000 pixel submillimeter camera that is due to be delivered shortly to the JCMT. The camera is expected to revolutionize submillimeter astronomy in terms of its ability to carry out wide-field surveys to unprecedented depths. SCUBA-2 uses transition-edge superconducting (TES) bolometer arrays, which come complete with in-focal-plane superconducting quantum interference device (SQUID) amplifiers and multiplexed readouts, and are cooled to $100 \mathrm{mK}$ by a liquid cryogen-free dilution refrigerator. SCUBA-2 will observe simultaneously at 850 and $450 \mu \mathrm{m}$, with angular resolutions of 14" and 8", respectively (Holland et al. 2006).

The polarimeter POL-2 will have an achromatic, continuously rotating half-wave plate in order to modulate the signal at a faster rate than atmospheric transparency fluctuations. Such a modulation should improve significantly the reliability and accuracy of submillimeter polarimetric measurements. The signal will be analyzed by a wire-grid polarizer. For calibration, a removable polarizer will also be available. The components are (in the order that the radiation will encounter them) the calibration polarizer, the rotating wave plate, and the polarizer. The components will be mounted in a box fixed permanently in front of the entrance window of the main cryostat of SCUBA-2. All components will be mounted so that they can be taken in and out of the beam remotely, making it very easy and fast to start polarimetry at the telescope (Bastien et al. 2005).

HARP is a $350 \mathrm{GHz}, 4 \times 4$ element heterodyne focal plane array that uses SIS detectors and was recently commissioned on the JCMT. Working in conjunction with the back-end AutoCorrelation Spectrometer and Imaging System (ACSIS; Hovey et al. 2000), HARP provides three-dimensional imaging capability with high sensitivity at 325 to $375 \mathrm{GHz}$. This is the first submillimeter spectral imaging system on JCMT, affording significantly improved productivity in terms of speed of mapping. The core specification for the array is that the combination of the receiver noise temperature and beam efficiency, weighted optimally across the array, is $<330 \mathrm{~K}$ single sideband (SSB) for the central $20 \mathrm{GHz}$ of the tuning range (Smith et al. 2003). The 16 pixels have receiver temperatures of 94-165 K. The angular resolution of HARP is $14^{\prime \prime}$, matching the $850 \mu \mathrm{m}$ resolution of SCUBA-2.

ACSIS has 16 inputs (actually 32, paired up), with a maximum bandwidth per channel of approximately $2 \mathrm{GHz}$ in a $2 \times 1 \mathrm{GHz}$ configuration. It has a minimum sample time of 
$50 \mathrm{~ms}$ and a maximum output map size of 16 Gbytes. It has a number of spectral bandwidths and resolutions that can be selected by the user: $250 \mathrm{MHz}$ bandwidth with $30 \mathrm{kHz}$ resolution, $500 \mathrm{MHz}$ bandwidth with $61 \mathrm{kHz}$ resolution (multisubsystem mode), $1 \mathrm{GHz}$ bandwidth with $500 \mathrm{kHz}$ resolution, and $2 \mathrm{GHz}$ bandwidth with $1000 \mathrm{kHz}$ resolution (merged). In practice, the usable bandwidth will be about $10 \%$ less than this, because of the filter roll-off.

ACSIS and HARP together have a number of observing modes, including raster mapping with position switching for mapping large areas, chopped jiggle mapping for fully sampled mapping of areas comparable to the HARP focal plane area, and jiggle mapping with fast frequency switching for fully sampled mapping of compact areas where no nearby off-source reference position is available.

During the planning phase of observations with the new JCMT instrumentation, numerous ideas were put forward for science questions that could be addressed (e.g., Ward-Thompson 2004). From these plans a number of proposals emerged. One such proposal, described herein, was to map local starforming regions with SCUBA-2, HARP, and POL-2. This was one of seven proposals accepted as part of the JCMT Legacy Program.

With the increased mapping speed of SCUBA-2, one can cover essentially all of the star-forming regions within $0.5 \mathrm{kpc}$ in a reasonable amount of time, detecting all of the protostars and prestellar cores. SCUBA-2 is designed to have increased sensitivity in each pixel, but also has 2 orders of magnitude more pixels than SCUBA, giving it about a thousand-fold increase in mapping speed. The increased mapping speed of SCUBA-2 can be illustrated by comparison with the large-scale mapping survey of the Galactic center carried out by SCUBA (Pierce-Price et al. 2000), which took $\sim 50 \mathrm{hr}$ of telescope time and covered only $1.4 \mathrm{deg}^{2}$. By contrast, this survey with SCUBA-2 will cover roughly $700 \mathrm{deg}^{2}$ to a greater depth in $\sim 120 \mathrm{hr}$.

We will use SCUBA-2 to map the submillimeter continuum emission from as many clouds within $0.5 \mathrm{kpc}$ as are visible from the JCMT, including several well-known Gould Belt clouds, such as Orion, Taurus, Perseus, and Ophiuchus. Several objects outside of the Gould Belt, including nearby Bok globules, will also be mapped (see, e.g., Launhardt et al. 1997). We estimate that the source catalog that we will produce will contain over 5000 sources. Such large samples of protostars and starless cores are required to provide robust statistics on objects over a range of evolutionary stages. With SCUBA-2's predicted improvement in per-pixel sensitivity over SCUBA, we will measure the prestellar clump mass functions down to substellar masses.

HARP increases the heterodyne mapping speed of the JCMT by over an order of magnitude. We will use this increased mapping speed to map a significant fraction of the SCUBA-2 sources in isotopologues of $\mathrm{CO}$. The combination of dust continuum maps from SCUBA-2 plus spectral-line data cubes from the heterodyne array at matched resolution will be extremely powerful. Since molecular clouds are highly turbulent and star formation generates infall, outflow, and rotational motions, velocity measurements are critical in understanding the massassembly process, feedback, and star formation efficiency (e.g., Goodwin et al. 2004a, 2004b; Vazquez-Semadeni et al. 2005). In addition, another use of the HARP data will be a determination of the amount of line contamination in the SCUBA data. Johnstone et al. (2003) considered this problem in Orion and argued that it was not significant, except for faint sources. With the increased sensitivity of SCUBA-2, such contamination may be important.

POL-2 will be able to make polarization maps of both highand low-density material in molecular clouds. We will use it to map 100 bright sources found in the SCUBA-2 survey. At present, there is a debate over the relative importance of magnetic fields and turbulence in regulating the star formation process (e.g., Mouschovias 1991; Padoan \& Nordlund 2002). Combined with kinematics from HARP, the POL-2 observations will allow for an investigation into the balance between gravity, turbulent support, and magnetic fields over a statistically meaningful number of star-forming cores. Previously, only a few cores have been mapped (e.g., Holland et al. 1999; Ward-Thompson et al. 2000; Matthews \& Wilson 2002; Crutcher et al. 2004; J. Kirk et al. 2006).

The goal of this paper is to outline the aims of the Gould Belt Survey and to describe the observations that will be carried out. In total, this program will take roughly $1000 \mathrm{hr}$ of observing time on the JCMT, 500 of which have been allocated over the first 2 years between 2007/2008 and 2009/2010. Section 2 details the SCUBA-2 aspects of the survey, $\S 3$ discusses the HARP survey, $\S 4$ describes the survey to be carried out with POL-2, $\S 5$ outlines some surveys at far-infrared wavelengths being carried out in parallel with this survey, and $\S 6$ provides a brief summary of the paper.

\section{SCUBA-2 SURVEY}

To obtain a complete view of the star formation in the Gould Belt, we need an inventory of all protostellar objects contained in these clouds. We will map with SCUBA-2 all of the starforming regions within $0.5 \mathrm{kpc}$ in the Gould Belt that are accessible to the JCMT. The sample, which includes many wellknown regions, will provide a very significant snapshot of star formation near the Sun. We will map the thermal dust emission at $850 \mu \mathrm{m}$ toward the $A_{V}>1$ areas of our target clouds (see Table 1) to a uniform depth, with a resolution of 14". In higher extinction regions $\left(A_{V}>3\right)$, we will go deeper and use better weather to observe at both 850 and $450 \mu \mathrm{m}$-the latter has a superior resolution of $8^{\prime \prime}$.

Table 1 lists details of the main clouds to be mapped, and Figures 3-13 illustrate the approximate mapping areas. This will provide a legacy of images, as well as point-source and extended-source catalogs, of roughly $700 \mathrm{deg}^{2}$ of sky. These 
TABLE 1

Technical Details of Approximate Areas to Be Mapped with SCUBA-2

\begin{tabular}{|c|c|c|c|c|c|c|}
\hline \multirow[b]{3}{*}{ REGION NAME } & \multicolumn{3}{|c|}{ Shallow-Survey Details } & \multicolumn{3}{|c|}{ Deep-Survey Details } \\
\hline & \multicolumn{2}{|c|}{ Field Center } & \multirow{2}{*}{$\begin{array}{c}\text { Area } \\
\left(\mathrm{deg}^{2}\right)\end{array}$} & \multicolumn{2}{|c|}{ Field Center } & \multirow{2}{*}{$\begin{array}{l}\text { Area } \\
\left(\mathrm{deg}^{2}\right)\end{array}$} \\
\hline & R.A. & Decl. & & R.A. & Decl. & \\
\hline Orion $\ldots \ldots \ldots \ldots$ & 054000 & -020000 & 81.1 & 054000 & -020000 & 14.3 \\
\hline Taurus & 043200 & +261000 & 45.5 & 043200 & +261000 & 4.5 \\
\hline Auriga . & 042000 & +380500 & 60.3 & 043100 & +364000 & 2.5 \\
\hline Perseus $\ldots \ldots \ldots$ & 033700 & +311500 & 15.5 & 033700 & +311500 & 2.3 \\
\hline Cepheus ........ & 212000 & +723000 & 37.7 & 212000 & +723000 & 1.0 \\
\hline IC $5146 \ldots \ldots \ldots$ & 024800 & +473000 & 0.8 & 024800 & +473000 & 0.5 \\
\hline Serpens $\ldots \ldots \ldots$ & 182300 & -031000 & 29.1 & 182300 & -031000 & 14.7 \\
\hline Pipe ............. & 173100 & -260000 & 16.4 & 173100 & -260000 & 7.2 \\
\hline Ophiuchus ...... & 162600 & -243000 & 30.4 & 162600 & -243000 & 6.5 \\
\hline Scorpius $\ldots \ldots \ldots$ & 165100 & -252000 & 31.6 & 165100 & -252000 & 1.4 \\
\hline Lupus I ......... & 154000 & -343000 & 23.3 & 154000 & -343000 & 3.5 \\
\hline Lupus II ......... & 160000 & -380000 & 1.7 & 160000 & -380000 & 0.2 \\
\hline Lupus $V$........ & 162000 & -373000 & 15.7 & 162000 & -373000 & 2.4 \\
\hline $\mathrm{CrA} \ldots \ldots \ldots \ldots$ & 191500 & -373000 & 11.0 & 190200 & -370000 & 2.8 \\
\hline
\end{tabular}

NoTE. - Units of right ascension (J2000.0) are hours, minutes, and seconds, and units of declination (J2000.0) are degrees, arcminutes, and arcseconds.

maps will be sensitive to every Class 0 and I protostar in the Gould Belt, and every L1544-like prestellar core within 200 pc (J. Kirk et al. 2005). The maps will yield the first extensive catalog of such objects selected by submillimeter

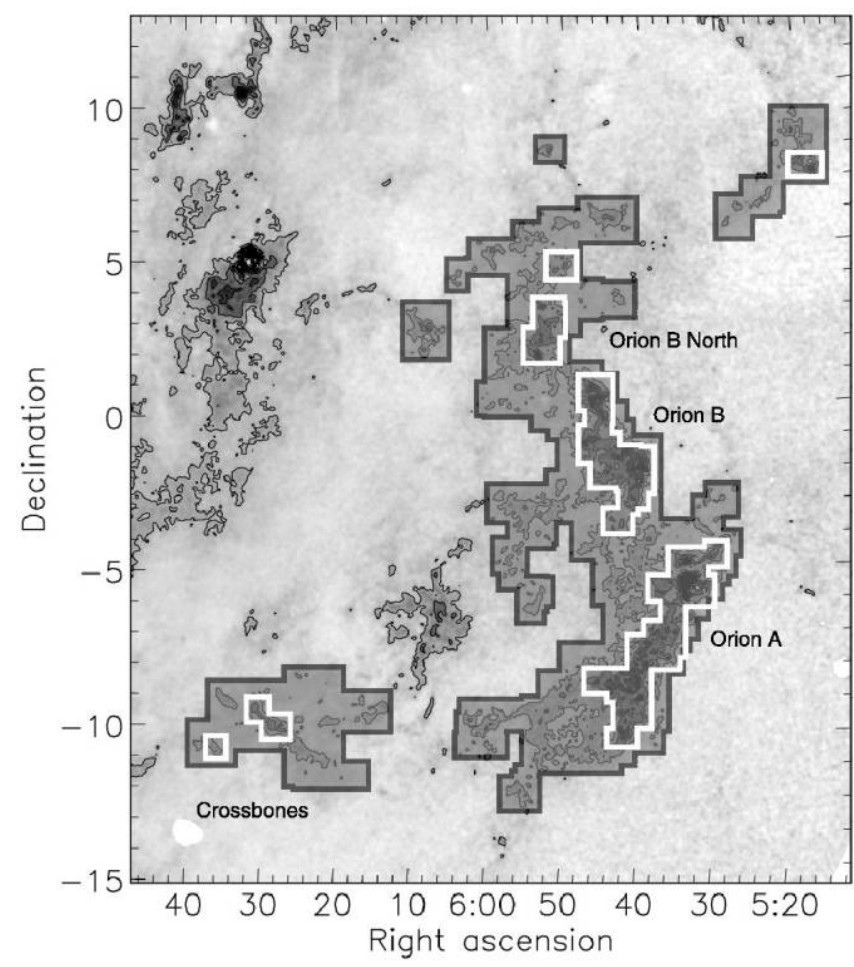

FIG. 3.-Extinction map of Orion, showing the approximate area planned to be mapped with SCUBA-2. The areas surrounded by the dark border will be mapped in the wide, shallow survey, and the areas surrounded by the white border will be mapped in the narrow, deep survey. continuum emission and will increase the number of known sources by more than an order of magnitude. Comparison with observations taken at other wavelengths, such as with Spitzer or Herschel, will allow correct classification of the sources.

The key science goals of the SCUBA-2 survey are:

1. To calculate the duration of each of the protostellar stages.

2. To elucidate the nature of the evolution of protostellar collapse.

3. To discover the origin of the IMF of stars, from intermediate-mass stars to substellar objects.

4. To discern the connection between protostars and the molecular cloud structure from which they formed.

In addition, the SCUBA-2 maps will provide "finding charts," both for the other aspects of this survey and for future

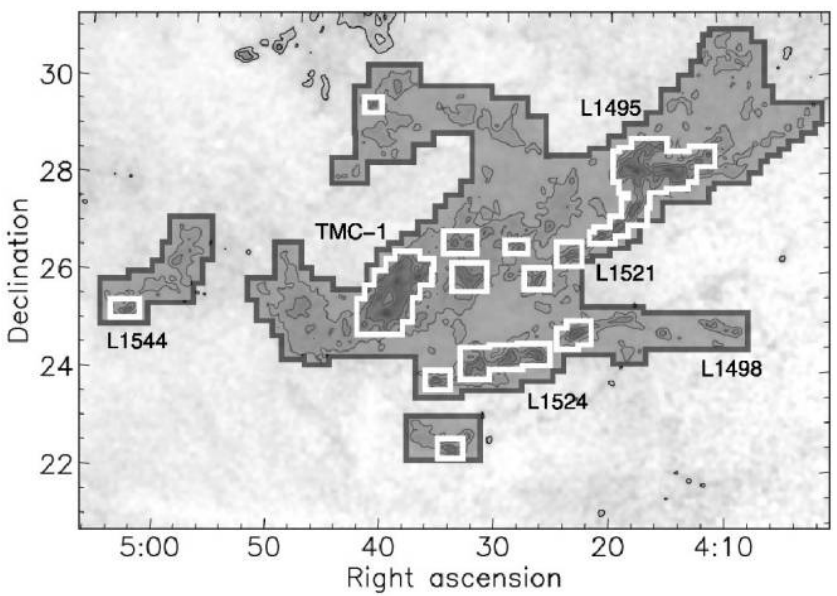

FIG. 4.-Extinction map of Taurus. Details as in Fig. 3. 


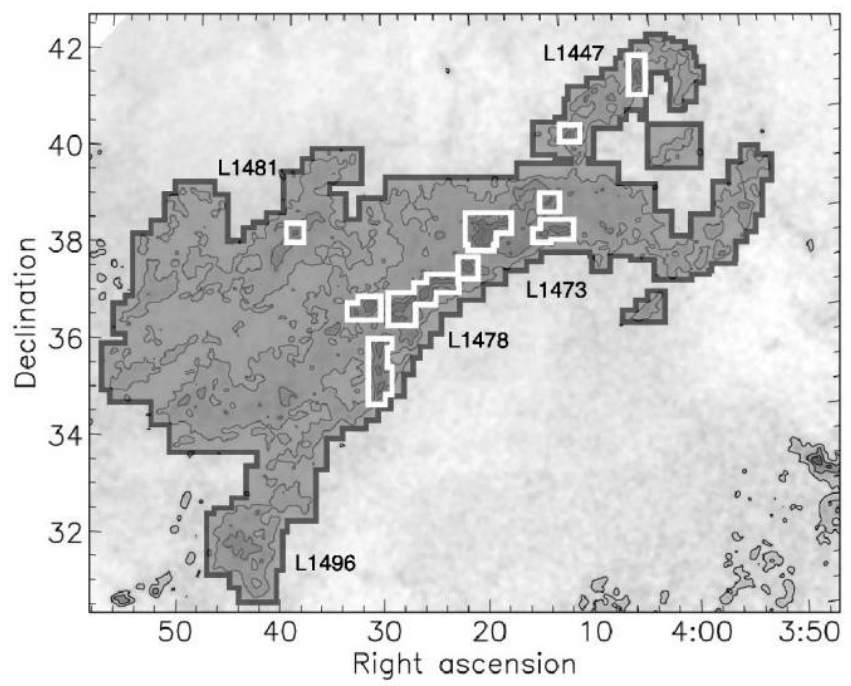

FIg. 5.-Extinction map of Auriga. Details as in Fig. 3.

projects. SCUBA-2 will take this subject beyond the sourceby-source approach of the past, into the domain where largenumber statistics on the earliest stages of star formation can finally be carried out, through a wide census of starless and prestellar cores and protostars.

To avoid mapping large areas of blank sky, molecular clouds have been preselected by visual extinction $A_{V}$ from the recent extinction atlas of Dobashi et al. (2005). The continuum mapping will be divided into two layers: a wide survey of areas with $A_{V}=1-3$, and a deep survey of areas with $A_{V}>3$. Figures 3-13 show the extents of each of the surveys in each region. In addition, some "blank field" areas will also be mapped as a control sample.

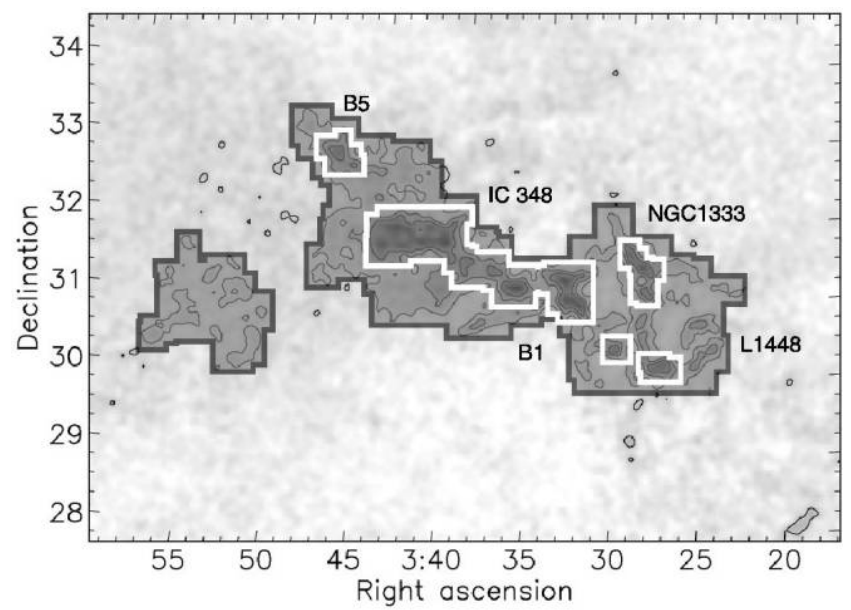

FIG. 6.-Extinction map of Perseus. Details as in Fig. 3.

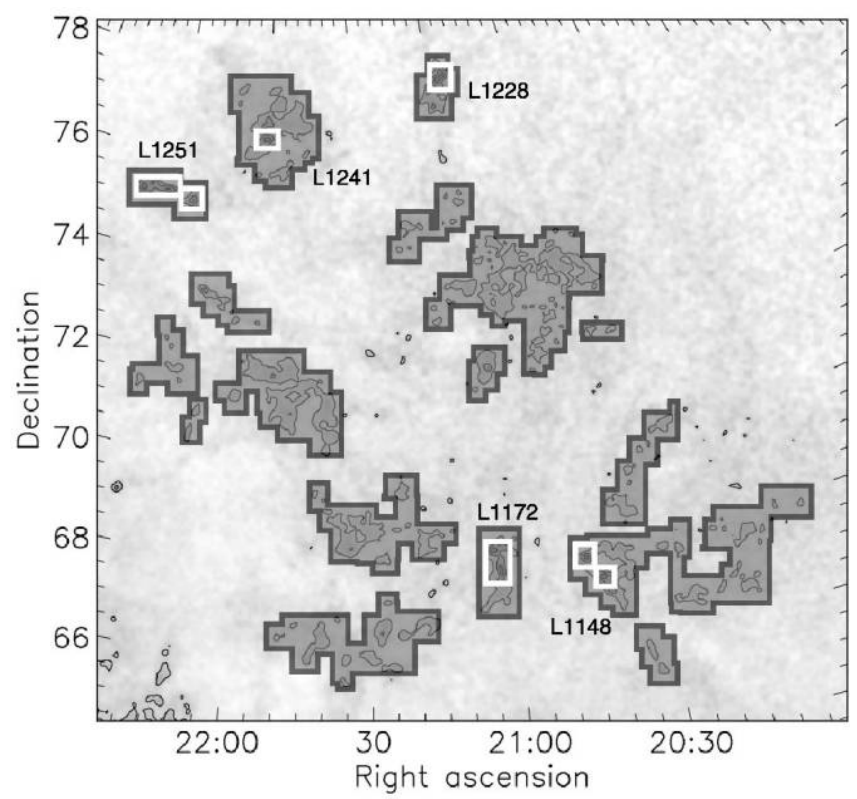

FIG. 7.-Extinction map of Cepheus. Details as in Fig. 3.

\subsection{Shallow Survey}

For the shallow survey, we will map areas with $A_{V}>1$ at $850 \mu \mathrm{m}$ to a depth of $1 \sigma=10 \mathrm{mJy}^{-1}$ beam $^{-1}$. Within the Gould Belt, this comprises an area of $\sim 400 \mathrm{deg}^{2}$. In addition, we will map $120 \mathrm{deg}^{2}$ that are outside of the major star-forming complexes but are positionally associated with the Gould Belt. This will cover nearby small clouds and isolated star formation regions selected from dark cloud catalogs (e.g., Lynds 1962; Cambrésy 1999; Dobashi et al. 2005) and previous catalogs

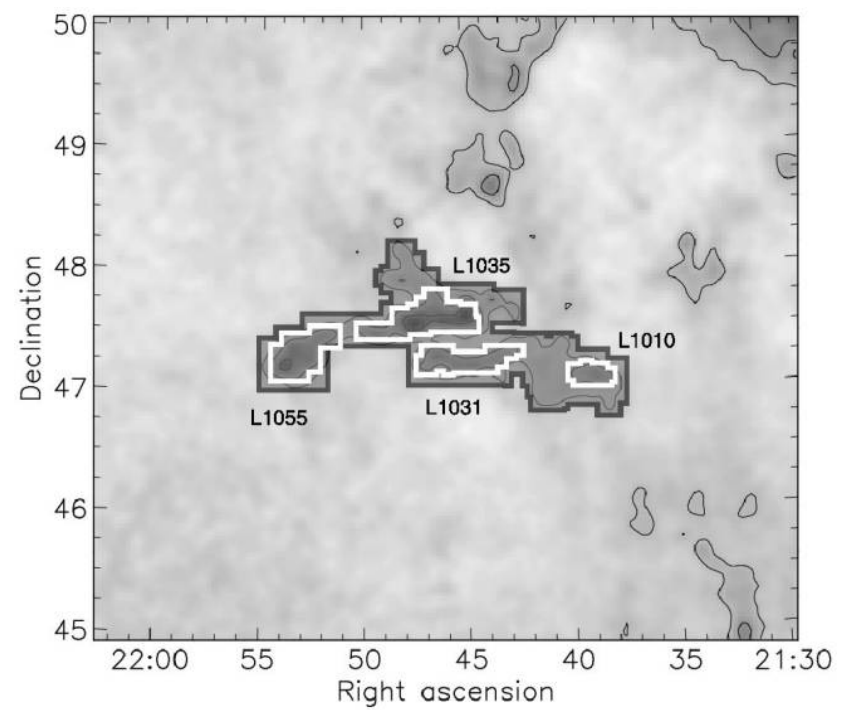

FIG. 8.-Extinction map of IC 5146. Details as in Fig. 3. 


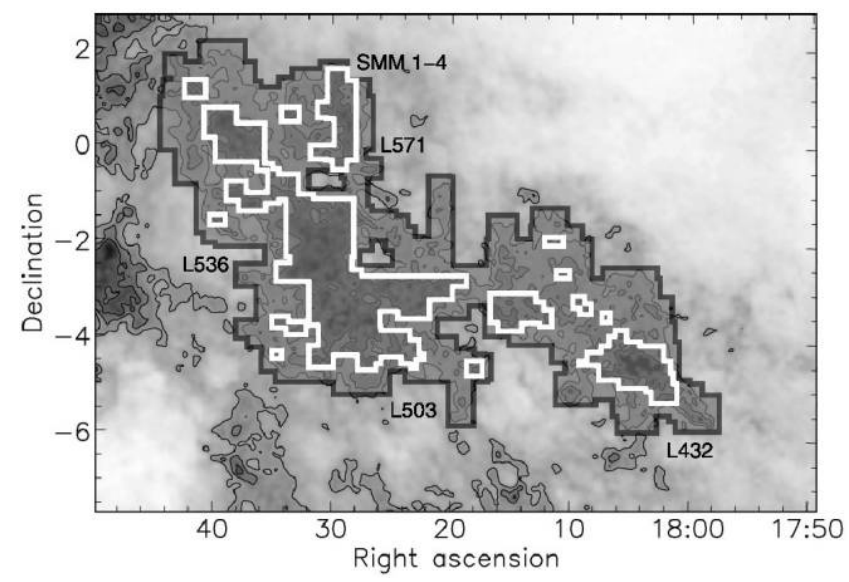

FIG. 9.-Extinction map of Serpens. Details as in Fig. 3.

(e.g., Clemens \& Barvainis 1988; Jijina et al. 1999; Lee \& Myers 1999; Visser et al. 2002).

Finally, we will map $\sim 10 \mathrm{deg}^{2}$ of blank sky (i.e., $A_{V}<1$ ) split into several fields near the Gould Belt, to the same depth. This will act as a control sample to see if we have missed significant numbers of objects by using $A_{V}$ to select our target regions. The data from the shallow survey will be sensitive at $3 \sigma$ to masses down to the substellar mass limit of $0.08 M_{\odot}$ per beam for objects at $0.5 \mathrm{kpc}$ that have $T_{d} \geq 20 \mathrm{~K}$, which is typical of the low-extinction parts of molecular clouds.

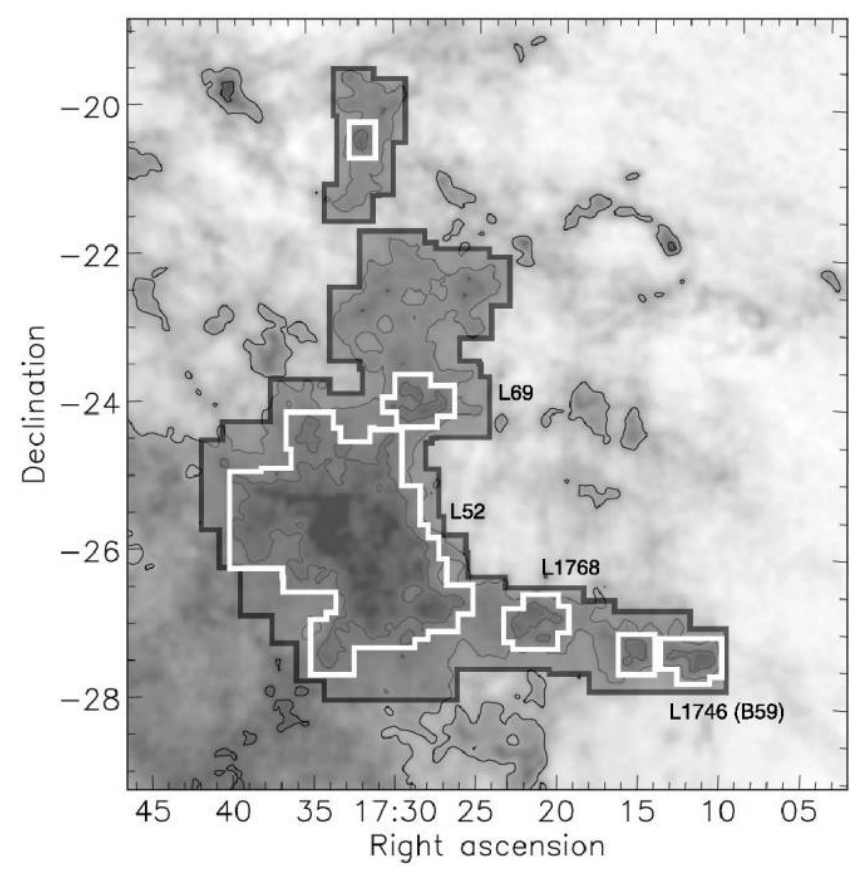

FIg. 10.-Extinction map of the Pipe Nebula. Details as in Fig. 3.

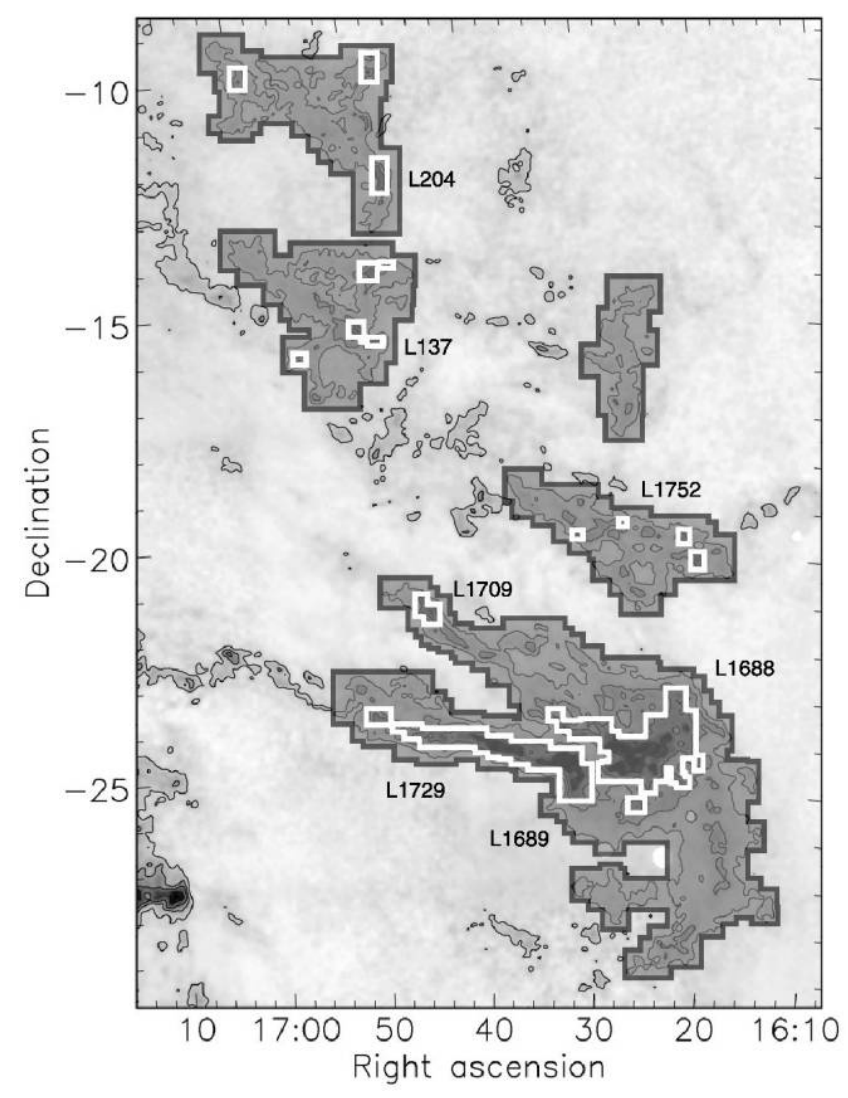

FIG. 11.-Extinction map of Oph-Sco. Details as in Fig. 3.

\subsection{Deep Survey}

Temperatures vary within molecular clouds, and the inner regions are colder $\left(T_{d} \sim 10 \mathrm{~K}\right)$ than the outer regions $\left(T_{d} \sim\right.$ $20 \mathrm{~K}$ ), due to increased shielding from the interstellar UV field. For the deep survey, we will map regions with $A_{V}>3$ to a

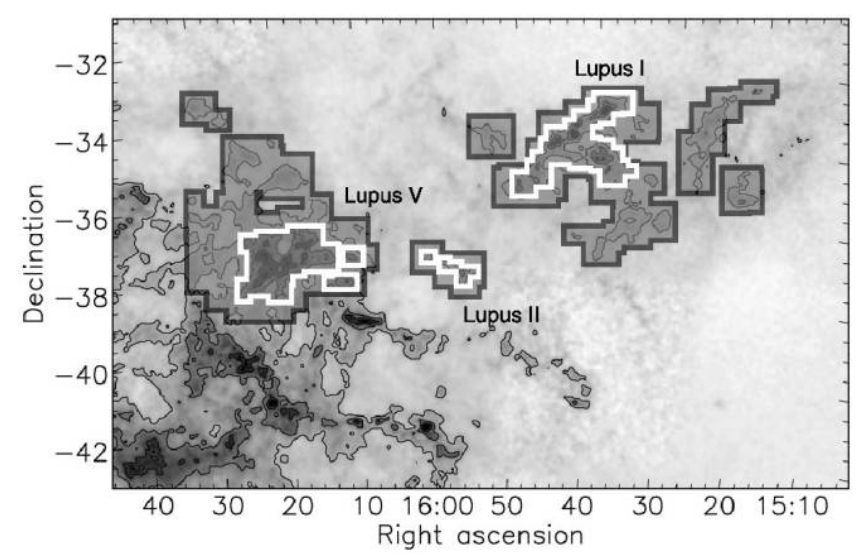

FIG. 12.-Extinction map of Lupus. Details as in Fig. 3. Note that Lupus III, IV, and VI are too far south to map with JCMT. 


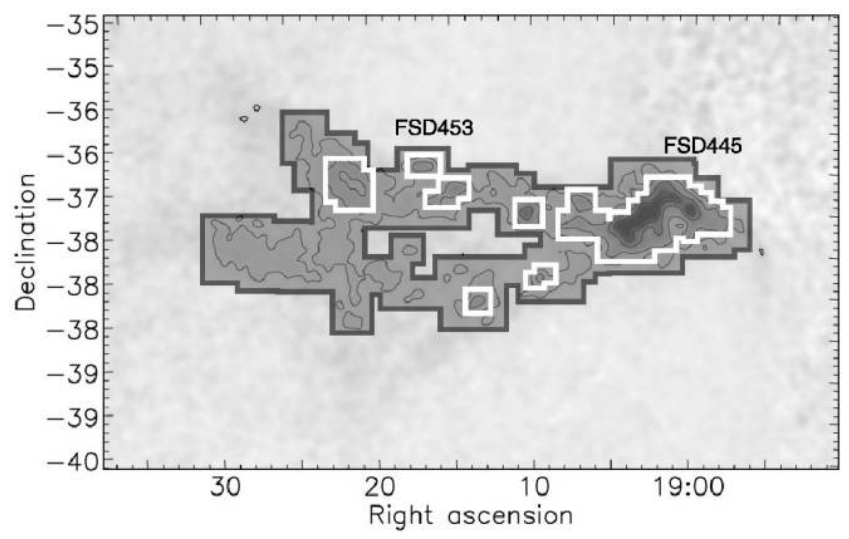

FIg. 13.-Extinction map of Corona Australis. Details as in Fig. 3.

depth of $1 \sigma=3 \mathrm{mJy}^{-1}$ beam $^{-1}$ at $850 \mu \mathrm{m}$ to ensure a complete census of star-forming cores. These regions comprise an area of $\sim 64 \mathrm{deg}^{2}$. These data will also reach the substellar mass limit of $0.08 M_{\odot}$ per beam at $3 \sigma$ for objects at $0.5 \mathrm{kpc}$ at $T_{d}=10 \mathrm{~K}$.

Furthermore, these observations will simultaneously provide maps at $450 \mu \mathrm{m}$ with a mean $1 \sigma \mathrm{rms}$ of $12 \mathrm{mJy}$ per $450 \mu \mathrm{m}$ beam (i.e., equal to $6 \mathrm{mJy}$ per $850 \mu \mathrm{m}$ beam after smoothing). This is because this aspect of the survey will be carried out in "grade 1" weather conditions $\left(\tau_{225 \mathrm{GHz}} \leq 0.05\right)$. The 450 and $850 \mu \mathrm{m}$ data together will provide spectral index information, where it is essential to have comparable resolution, to constrain the dust opacity indices and thus the masses of the objects.

\subsection{Source Count Predictions}

The total star formation rate for clouds within $0.5 \mathrm{kpc}$ is $\sim 6 \times 10^{-3} M_{\odot} \mathrm{yr}^{-1}$ (e.g., McKee \& Williams 1997). Using the measured IMF (e.g., Kroupa 2001), the total stellar production rate within this distance is therefore $\sim 0.02 \mathrm{stars}^{\mathrm{yr}^{-1}}$. The best current estimates of the timescales for prestellar cores and Class 0 protostars are $\sim 3 \times 10^{5}$ and $\sim 3 \times 10^{4} \mathrm{yr}$, respectively (e.g., André et al. 2000). Thus, we expect $\sim 6000$ prestellar cores and $\sim 600$ Class 0 protostars to be found in our wide survey. Even allowing for possible uncertainties in these by factors of $\sim 2$, we would still expect thousands of objects in total, with hundreds at low mass $\left(M<0.5 M_{\odot}\right)$ and tens at high mass $\left(M>8 M_{\odot}\right)$.

These objects will fill in the underpopulated extremes of currently measured mass functions. Note that only tens of Class 0 protostars and hundreds of prestellar cores in total are currently known (André et al. 2000). Finally, the expected numbers of objects at the mass function peak $\left(M \approx 0.5 M_{\odot}\right)$ will be large enough ( $\sim 20-50$ in each cloud) to reveal statistically whether differences in characteristic stellar masses that exist between clouds are caused by local environmental influences on core formation, such as cloud density and sound speed.

\subsection{Protostellar Lifetimes and Accretion Rates}

The census of prestellar cores and protostars from the continuum mapping will allow us to calculate the relative duration of these stages. Since half of the envelope mass is accreted during the Class 0 stage, and the rest during the Class I stage (André et al. 1993), the duration of each stage tells us about the protostellar accretion rate. For instance, if they are roughly equal, then the accretion rate is probably constant, as in the Shu collapse model (Shu 1977).

Alternatively, if the Class 0 duration is only one-tenth of the Class I duration, as is currently suspected (André et al. 2000), then accretion must start very rapidly and decrease over time (e.g., Whitworth \& Ward-Thompson 2001), implying a very different collapse scenario. In addition, if much of the envelope mass is ejected (e.g., Matzner \& McKee 2000), then the accreted fractions will sum to less than 1. Current observations are limited by small-number statistics (e.g., Visser et al. 2002), but the wide survey will provide a sufficiently large sample to answer this question.

Furthermore, the relative quantities of prestellar cores at varying degrees of central condensation (given by continuum radial profiles) will inform models that predict the onset of protostellar collapse (e.g., turbulent dissipation vs. magnetic regulation).

\subsection{Origin of the IMF}

With the census of nearby prestellar cores provided by the continuum mapping, we will be able to plot a very well-populated mass spectrum of these objects over a very wide range of masses. This will allow us to confirm or refute the claim that this mass function (e.g., Motte et al. 2001; Johnstone et al. 2006; Nutter \& Ward-Thompson 2007) mimics the stellar IMF (Salpeter 1955). Recent observations appear to show that the prestellar core mass function follows the same form as the IMF down to very low masses (see Fig. 14) (Nutter \& WardThompson 2007).

Such a steep mass function implies that low-mass cores dominate (both by number and by mass). This is in contrast to the cloud mass function, where most of the mass resides in the largest structures (e.g., Williams et al. 1994), implying that a different physical mechanism is responsible for the cores. Two other obvious differences between the cloud and core mass function also exist. First, while the cloud mass function includes all the mass in the cloud (by definition), the core mass function generally only adds up to a few percent of the mass of the parent cloud (Johnstone et al. 2004; H. Kirk et al. 2006). Second, the mass-radius relation for clouds implies nonthermal motions for the largest structures, whereas the cores reveal mostly thermal motions.

If the link between the core mass function (CMF) and the IMF is confirmed, this will provide very strong evidence that the IMF is determined at the prestellar core stage of star formation; i.e., the physics behind core formation is also the cause 


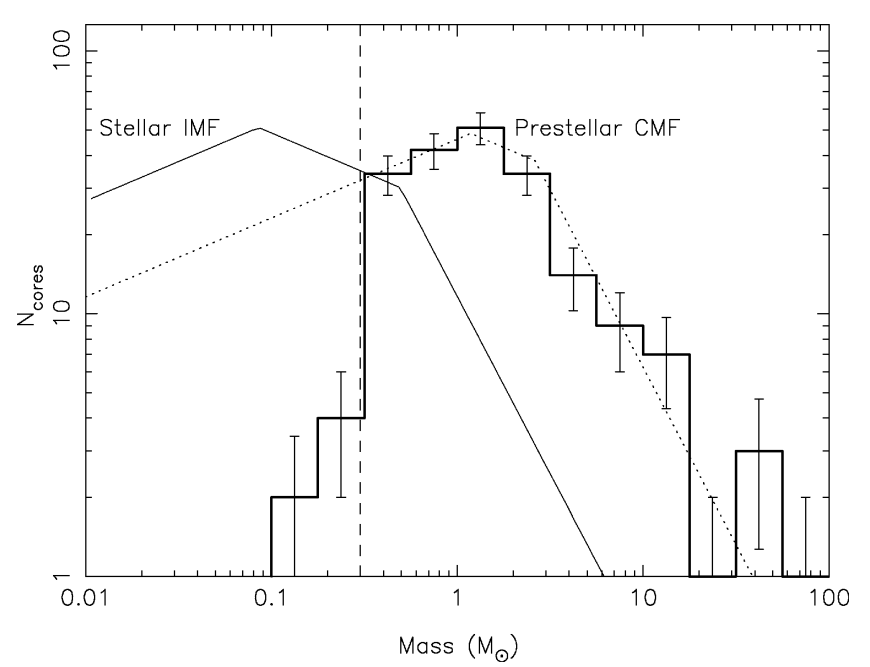

FIG. 14.-Core mass function (CMF) in Orion, from Nutter \& Ward-Thompson (2006). A three-part stellar IMF, normalized to the peak of the CMF, is overlaid as a thin solid line. The dotted line shows a three-part mass function, with the same slopes as the IMF, superimposed on the CMF. The agreement between the two appears to indicate that the stellar IMF is determined by the CMF.

of the IMF. The two currently competing theories of core formation invoke either magnetic fields or turbulence (e.g., Mouschovias 1991; Ballesteros-Paredes et al. 2003). Of particular note will be the detection of numerous objects with masses below the substellar limit $\left(0.08 M_{\odot}\right)$, allowing for the first time a comparison between the mass functions of very low mass molecular cloud cores and the brown dwarf IMF. Some detections of substellar mass cores have already been made (e.g., Greaves et al. 2003), but the numbers are very limited so far. Similarities between these mass functions would imply a similar formation process for brown dwarfs and stars. However, a statistically significant deviation would imply differing origins; for example, brown dwarfs may form out of protostellar disks (for a review, see Whitworth et al. 2007).

Additional important questions for these very low mass cores include whether they resemble the solar-mass cores, or exist only inside a collapsing region; whether they also resemble Bonnor-Ebert spheres, and if so, what provides the confining pressure; or alternatively, whether they only form within protostellar disks (e.g., Matzner \& Levin 2005). Thus, the survey has a capacity to constrain brown dwarf formation directly, as well as through IMF/CMF comparisons.

\subsection{Structure of Cores to Clouds}

With a distance limit of $0.5 \mathrm{kpc}$, the linear resolution of the continuum mapping at $850 \mu \mathrm{m}$ will be $0.03 \mathrm{pc}$ (7000 AU) or better. This scale is well matched for probing the structures of the detected prestellar and protostellar envelopes, as well as their surrounding environments. For example, the radial density profiles of prestellar cores show a flat inner region and steep outer region with the turnover at $\sim 0.03 \mathrm{pc}$ (Ward-Thompson et al. 1994; J. Kirk et al. 2005).

Some have claimed that the observed radial density profiles indicate that cores are pressure-supported Bonnor-Ebert spheres (e.g., Alves et al. 2001), while others have argued that such configurations result naturally from dynamic evolution of gas on large scales and do not indicate equilibrium (BallesterosParedes et al. 2003). The differences between the model predictions center around the velocity profiles of the cores. The larger sample of cores revealed by the continuum mapping and followed up by CO spectroscopy with HARP (see $\S 3$ below) will provide enough examples of both velocity structure and detailed morphology to settle this debate. On slightly larger scales, the continuum mapping will have the sensitivity to probe the lower density surroundings of these cores, allowing insights into core formation. For instance, if one assumes that the continuum maps trace the mass, then one could use them to assess the importance of gravitational torques on assembling disks (Jappsen \& Klessen 2004).

Previously, these surroundings could be probed only by lower density tracers, such as CO (in regions where it is not depleted and its lines are optically thin), but with continuum mapping, it will be possible to link the structures of cores with their environments, using the same tracer. On even larger scales, the continuum mapping (especially that of the deep survey) will reveal the structure of extended filaments within clouds (see, e.g., Johnstone \& Bally 1999; Fiege et al. 2004; Hatchell et al. 2005).

Molecular clouds exhibit filamentary structures, which are often the locus of star formation along their length. Although turbulence and magnetic fields have been suggested to explain the filamentary structures in molecular clouds, few detailed studies have been made, due to limitations in mapping both the velocity fields and the polarized light that traces the magnetic field. Coupled with the dynamical information obtained with HARP and the magnetic field geometry obtained with POL-2, the morphologies and structures of filaments revealed by the continuum mapping will provide clues to both the origins and evolution of molecular clouds (e.g., Khalil et al. 2004).

\section{HARP SURVEY}

In addition to the SCUBA-2 survey of the local molecular cloud population, we will also carry out a $\mathrm{CO}$ survey with HARP to trace the kinematics of cores and clusters, at the same spatial resolution as the $850 \mu \mathrm{m}$ SCUBA-2 observations of dust emission $\left(14^{\prime \prime}\right)$. This will allow us to address a large number of fundamental scientific problems in star formation in samples that for the first time are of statistically significant size.

In typical star-forming molecular cloud cores, temperatures and densities are in the ranges $10-50 \mathrm{~K}$ and $10^{4}-10^{5} \mathrm{~cm}^{-3}$, respectively, which are the conditions under which the $\mathrm{CO}$ and isotopic lines in the $350 \mathrm{GHz}$ range are excited (see Table 2).

The key goals of the line observations are: 
TABLE 2

CO IsOTOPOLOGUes to Be OBSERVED WITH HARP

\begin{tabular}{cccccc}
\hline \hline Transition & $\begin{array}{c}\nu \\
(\mathrm{GHz})\end{array}$ & $\begin{array}{c}E_{U} / k \\
(\mathrm{~K})\end{array}$ & $\begin{array}{c}\Delta v \\
\left(\mathrm{~km} \mathrm{~s}^{-1}\right)\end{array}$ & $\begin{array}{c}T_{\mathrm{rms}} \\
(\mathrm{K})\end{array}$ & $\begin{array}{c}N_{\mathrm{H}_{2}}(3 \sigma) \\
\left(\mathrm{cm}^{-2}\right)\end{array}$ \\
\hline${ }^{12} \mathrm{CO} J=3-2 \ldots \ldots$ & 345.7960 & 33.2 & 1.0 & 0.3 & $4.5 \times 10^{18}$ \\
${ }^{13} \mathrm{CO} J=3-2 \ldots \ldots$ & 330.5880 & 31.7 & 0.1 & 0.25 & $3.6 \times 10^{19}$ \\
$\mathrm{C}^{18} \mathrm{O} J=3-2 \ldots \ldots$ & 329.3305 & 31.6 & 0.1 & 0.3 & $3.3 \times 10^{20}$ \\
\hline
\end{tabular}

Notes. - Column density calculations assume LTE at $50 \mathrm{~K}$ for ${ }^{12} \mathrm{CO}$ and $20 \mathrm{~K}$ for ${ }^{13} \mathrm{CO}$ and $\mathrm{C}^{18} \mathrm{O}$. Abundances relative to $\mathrm{H}_{2}$ are taken to be $10^{-4}$ for ${ }^{12} \mathrm{CO}$ and $1.7 \times 10^{-7}$ for $\mathrm{C}^{18} \mathrm{O}$ (Frerking et al. 1982). The ratio of ${ }^{13} \mathrm{CO} / \mathrm{C}^{18} \mathrm{O}$ is assumed to be 77 (Wilson \& Rood 1994). The standard conversion of column density to visual extinction is taken such that an $A_{V}$ of 1 corresponds to a column density of $0.9 \times 10^{21}\left(N_{\mathrm{H}}+2 N_{\mathrm{H}_{2}}\right) \mathrm{cm}^{-2}$.

1. To search for and map any high-velocity outflows present in the cores in order to differentiate between starless and protostellar cores.

2. To derive simple constraints on the column density and $\mathrm{CO}$ depletion in these cores.

3. To help understand the support mechanisms and core evolution.

4. To characterize the cloud kinematics in a large sample of environments and investigate the evolution and role of turbulence in star formation.

We note that these goals are also very closely coupled to those of the polarimetric study presented below, and that many of the goals of the continuum survey also require these data for interpretation, particularly to classify and determine the ages of the embedded sources.

\subsection{Target Regions}

HARP is a much faster mapping instrument than previous single-pixel receivers. However, it is still not possible to map all of the regions covered by the SCUBA-2 survey. Hence, we will select a typical sample of cores and cloud regions from the SCUBA-2 survey and observe these with HARP.

The proposed molecular line observations consist of two sets of targets. We will make single, fully sampled footprint images $\left(\sim 4 \operatorname{arcmin}^{2}\right)$ of a set of 1000 cores, and larger $\left(0.08 \mathrm{deg}^{2}\right)$ maps of 10 cloud regions containing filaments and clustered star formation. For the well-studied clouds, many of the target regions are already known from previous SCUBA surveys. The cores, selected from the SCUBA-2 catalog, will consist of a flux-limited sample plus a representative selection covering the full range of conditions and clouds.

In order to meet the science goals outlined above, we will map all of the cloud regions and 300 of the cores in the ${ }^{12} \mathrm{CO}$, $\mathrm{C}^{18} \mathrm{O}$, and ${ }^{13} \mathrm{CO} J=3-2$ lines-the latter two lines can be obtained simultaneously within the ACSIS bandwidth. A further 700 cores will be mapped in the ${ }^{12} \mathrm{CO} 3-2$ line only. Most of the core maps will be a single, fully sampled HARP footprint, but for clustered cores, we will combine footprints to map contiguous areas.

As summarized in Table 2, the ${ }^{12} \mathrm{CO}$ maps will be made with

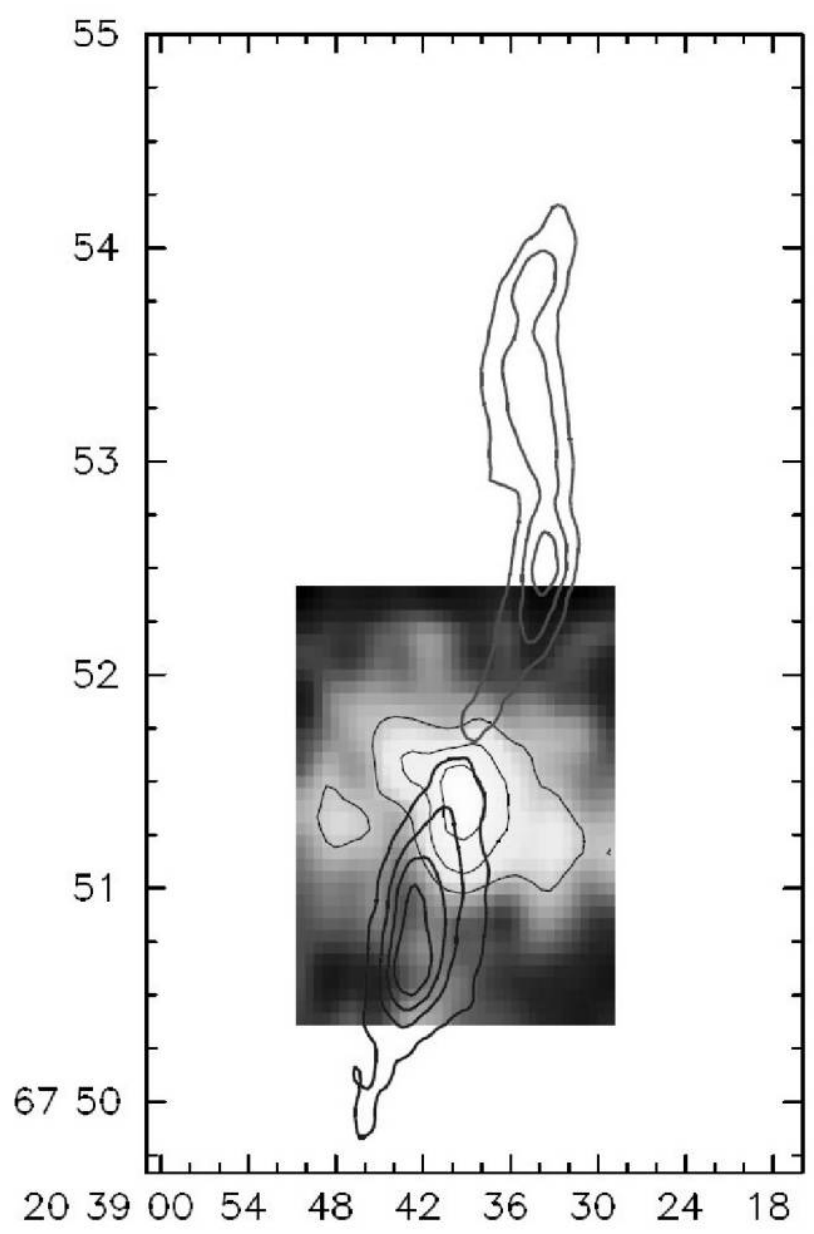

FIG. 15.-Class 0 source L1157 mapped in $\mathrm{C}^{18} \mathrm{O} 3-2$ (gray scale and thin contours) and ${ }^{12} \mathrm{CO} 3-2$ (thick contours).

a channel width of $1.0 \mathrm{~km} \mathrm{~s}^{-1}$. The target rms $1 \sigma$ noise level will be $0.3 \mathrm{~K}$ in each channel. The ${ }^{13} \mathrm{CO}$ and $\mathrm{C}^{18} \mathrm{O}$ maps will both have a channel width of $0.1 \mathrm{~km} \mathrm{~s}^{-1}$, and the target rms $1 \sigma$ noise level in each channel will be 0.25 and $0.3 \mathrm{~K}$, respectively. The slightly poorer predicted $\mathrm{rms}$ in $\mathrm{C}^{18} \mathrm{O}$ is due to its location near the edge of an atmospheric band. The equivalent $\mathrm{H}_{2}$ column density sensitivities are noted in Table 2 .

\subsection{Molecular Outflows: Classification and Mass Ejection}

The first observation targeting cores will be in the ${ }^{12} \mathrm{CO} J=$ 3-2 line to search for and image any high-velocity gas (see Fig. 15). The presence of a high-velocity molecular outflow is a standard way to differentiate between prestellar and protostellar cores (e.g., Bontemps et al. 1996b; Wolf-Chase et al. 1998; Visser et al. 2002; Hatchell et al. 2007). For compact submillimeter objects with no IR detection, outflows are a critical discriminant between prestellar and protostellar cores. Where outflows are detected, we will estimate outflow mo- 
mentum flux (e.g., Bontemps et al. 1996a) in this large, homogeneous sample, allowing us to put on a firm statistical footing the proposed correlations with bolometric luminosity and envelope mass.

The envelope mass-luminosity-momentum flux relationships test models of mass accretion and outflow ejection, and therefore how much of the envelope mass is ultimately converted into stars (Matzner \& McKee 2000). Reasonable estimates of momentum flux can be made without mapping the whole outflow, as most of the momentum flux is seen close to the star (Fuller \& Ladd 2002).

In the larger cloud regions, mapping the outflows of a large number of protostellar objects will make it possible to determine their energy input to the cloud (e.g., Norman \& Silk 1980; Li \& Nakamura 2006; Matzner 2007). The magnitudes of these energies will allow determination of the roles of outflows in stimulating turbulent motions or parent cloud dispersal (e.g., Silk 1995).

\subsection{CO Depletion}

We note that all molecular tracers are affected by chemistry and depletion onto dust grains. CO is no exception, depleting in the densest regions of molecular cores (e.g., Caselli et al. 1999; Redman et al. 2002). We will use these observations to investigate $\mathrm{CO}$ depletion.

Prestellar cores are known to be heavily affected, with $\mathrm{CO}$ depletion factors reaching 10 or more (e.g., Crapsi et al. 2005). A limited study suggests that Class 0 sources are also centrally depleted by factors of 5 , but by the time the Class I phase is reached, $\mathrm{CO}$ abundances have returned to the canonical value of $10^{-4}$ (Jorgensen et al. 2002).

Typical size scales for depletion are $\sim 6000$ AU (Caselli et al. 1999), which corresponds to $12^{\prime \prime}$ at $500 \mathrm{pc}$ or $40^{\prime \prime}$ at 150 pc. Outside the density peaks, CO abundance should be stable (e.g., van Dishoeck 2006; Di Francesco et al. 2007).

This survey will produce simultaneous dust and CO isotopologue images of hundreds of cloud cores, classified on the basis of infrared counterparts or molecular outflows. We can use this sample to statistically investigate the levels and size scales for $\mathrm{CO}$ depletion as a function of core properties such as mass and evolution.

\subsection{Fundamental Properties of Cores: Mass, Temperature, and Density}

Two independent measurements of the envelope gas mass, in order to compare with the dust mass, can be derived from $\mathrm{C}^{18} \mathrm{O}$. First, the $\mathrm{C}^{18} \mathrm{O}$ integrated line strength, assuming optically thin emission, will yield a gas mass. Although this will be a lower limit due to depletion, it is still valuable, as it relies on a different set of assumptions ( $\mathrm{CO}$ abundance, gas excitation) to mass estimates from dust, where the dust opacity and dust temperature are subject to their own uncertainties. Second, the velocity dispersion and size provide a virial mass estimate that is largely unaffected by depletion as line widths fall toward cloud cores (e.g., Goodman et al. 1998a, 1998b).

In total, the $\mathrm{CO}$ observations are vital to deriving reliable core parameters in order to understand how clusters form and how the initial mass function is determined. Combining the HARP data with existing lower $J$ maps of these clouds (e.g., Ridge et al. 2006) will allow us to estimate temperature and density using multitransition, multi-isotopologue radiative transfer methods.

\subsection{Kinematic Tests of Star Formation Models}

Measurement of the detailed kinematic and density properties of the cores will be carried out using the $\mathrm{C}^{18} \mathrm{O}$ and ${ }^{13} \mathrm{CO}$ lines, observable simultaneously (see Fig. 15). In nearly all cores, we expect the $\mathrm{C}^{18} \mathrm{O}$ line to be optically thin. Hence, we will be able to measure the thermal and nonthermal contributions to the line width, enabling a study of core support mechanisms and their evolution (e.g., Jessop \& Ward-Thompson 2001).

The combination of a high-density tracer, such as the $J=$ 3-2 transition, with lower density tracers (e.g., lower $J$ CO lines) allows an investigation of the dynamical motion of cores through their parent cloud (e.g., Walsh et al. 2004, 2006; Ayliffe et al. 2007).

Where cores are clustered, we will also compare the velocity dispersion between cores, a simple measurable quantity that can be compared with both star formation simulations and young clusters of pre-main-sequence stars (e.g., Belloche et al. 2001).

These quantities may potentially discriminate between competitive accretion/core mergers (e.g., Bate et al. 2003) and the alternative, where one submillimeter core results in one star system (e.g., Goodwin et al. 2004a, 2004b).

In addition, the distribution of $\mathrm{C}^{18} \mathrm{O}$ line velocities of cores observed with HARP in individual molecular clouds will probe theories of cloud turbulence. Such cores may act as test particles within the larger scale turbulent motions within such clouds. Similar smaller scale studies have recently been carried out in, for example, Perseus (H. Kirk et al. 2007).

\subsection{Clouds and Filaments}

We will also produce large maps of 10 filamentary or clustered star formation regions. Many of these will be well-studied regions (e.g., the Orion filament and the $\rho$ Ophiuchi cluster), but not all of them have preexisting submillimeter maps (e.g., the Pipe Nebula and L1506 in Taurus). These regions will be mapped in ${ }^{12} \mathrm{CO}, \mathrm{C}^{18} \mathrm{O}$, and ${ }^{13} \mathrm{CO}$ lines to the spectral resolutions summarized in Table 2. These maps will cover regions of an order of $300 \operatorname{arcmin}^{2}\left(0.08 \mathrm{deg}^{2}\right)$ in size.

The key goal here will be to compare the observations to gas dynamic and magnetohydrodynamic (MHD) turbulence simulations (e.g., Balsara et al. 2001; Padoan \& Nordlund 2002; Bate et al. 2003; Vazquez-Semadeni et al. 2005). These models 
make strong predictions on the expected clump mass spectrum, the spatial and velocity structure, the shapes of the cores and filaments, and the star formation efficiency.

Comparisons can be made by analyzing cloud spatial and velocity structure, using techniques such as clumping analyses (Williams et al. 1994), axial ratios (e.g., Kerton et al. 2003), delta variance (Stutzki et al. 1998; Bensch et al. 2001), principal component analysis (e.g., Heyer \& Schloerb 1997), structure function (e.g., Brunt et al. 2003), and spectral correlation function (Rosolowsky et al. 1999).

Mapping the $J=3-2$ transitions, which trace reasonably high densities, at an angular resolution typically less than a thermal Jeans length (for an $\mathrm{H}_{2}$ number density of $10^{5} \mathrm{~cm}^{-3}$ ) reveals the kinematics over the critical transition to gravitational dominance (Ossenkopf 2002), whereas existing low- $J$ CO maps (e.g., Dame et al. 2001; Wilson et al. 2005) trace only the lower density large-scale molecular cloud structure and kinematics. We note that lower $J$ (usually $J=1-0$ ) maps exist for many of our clouds and can be analyzed in conjunction with the HARP data (e.g., Ridge et al. 2006).

In total, the $\mathrm{CO}$ observations are vital in deriving reliable core parameters in order to understand how clusters form and how the initial mass function is determined. When combined with the SCUBA-2 data, the HARP data will provide a powerful way to explore the detailed dynamics and density structure in star-forming clouds, presenting rigorous tests of the details of the theoretical models.

\section{POL-2 SURVEY}

We will also carry out a polarimetric survey of the brightest cores and clouds, since measurements of polarized emission from dust are the most effective means of probing the magnetic field. By contrast, absorption polarimetry is limited to the periphery of dense clouds, and Zeeman splitting detections are made in only a small fraction of observed regions (Crutcher 1999).

Polarized dust emission is detected from objects on all measured scales (e.g., Matthews et al. 2001; Crutcher et al. 2004) and from compact cores, regardless of evolutionary epoch (e.g., Ward-Thompson et al. 2000; Matthews \& Wilson 2002), as illustrated in Figure 16. Sensitivity limitations and practical limits on observing times have previously restricted observations to several dozen bright (i.e., $S_{850 \mu \mathrm{m}} \geq 1 \mathrm{Jy}$ ), compact objects.

Attempts to measure the magnetic field in star-forming regions are driven by the need to understand its significance in the formation of cloud structure and/or the regulation of collapse of cloud cores. These factors are related to star formation rates and molecular cloud lifetimes, on which there is substantial debate in the literature (e.g., Myers \& Goodman 1988; Hartmann et al. 2001; Elmegreen 2000).

The key goals of the polarimetric mapping are:
1. To obtain maps of polarization position angle and fractional polarization in a statistically meaningful sample of cores.

2. To characterize the evidence for and relevance of the field and turbulence (in conjunction with HARP observations) in cores and their surrounding environments.

3. To test the predictions of low-mass star formation theories (core, outflow, field geometry).

4. To generate a large sample of cores that are suitable for follow-up with forthcoming instruments, such as ALMA.

\subsection{Target Regions}

From the SCUBA-2 $850 \mu \mathrm{m}$ continuum survey, we expect to detect at least 100 cores of sufficient brightness $(>250 \mathrm{mJy}$ beam $^{-1}$ peak $850 \mu \mathrm{m}$ flux) for observation with POL-2 in single SCUBA-2 footprints. These polarimetry targets will be located in different star-forming regions and be of various classes (starless, prestellar, Class 0, Class I).

To probe the initial field conditions for core formation, maps of $\sim 0.08 \mathrm{deg}^{2}$ in extent of 10 cloud regions containing filaments and clustered star formation will be made in synergy with the HARP large-scale mapping to measure the gas dynamics and characterize the turbulence.

Candidate types of regions would include a starless fragment of a filamentary cloud, such as a portion of the Pipe Nebula (see Fig. 10). In addition, we will map filamentary clouds for which absorption polarimetry exists, such as the L1506 filament in Taurus. We will use these data to directly compare the magnetic field directions yielded by emission and absorption polarimetry at the same locations, allowing these methods to be directly compared for the first time. We will also map regions around clusters exhibiting different rates of star formation.

\subsection{Tests of the Models}

According to one paradigm of low-mass star formation, collapse is guided by magnetic fields, producing flattened cores and disks (e.g., Mouschovias 1991). Outflows are then generated orthogonal to the disk, producing outflows aligned with the nascent field direction. Greaves et al. (1997) tested this correlation for five sources, finding that the alignment of field and outflow appeared to be correlated to the angle of the outflow to the line of sight (see also Matthews et al. 2007).

With $\sim 70$ sources with outflows (Class 0 and Class I), we will be able to establish statistically whether outflows are preferentially oriented with respect to the field direction. Using the SCUBA-2 data, we will also determine whether the field direction is related to the core morphology. Many cores for which polarization maps exist are too distant to compare field geometry to core morphology, since the cores are poorly resolved. A sizeable fraction of the objects that are mapped are also embedded in filaments, making measurements of core axis ratios particularly challenging. 

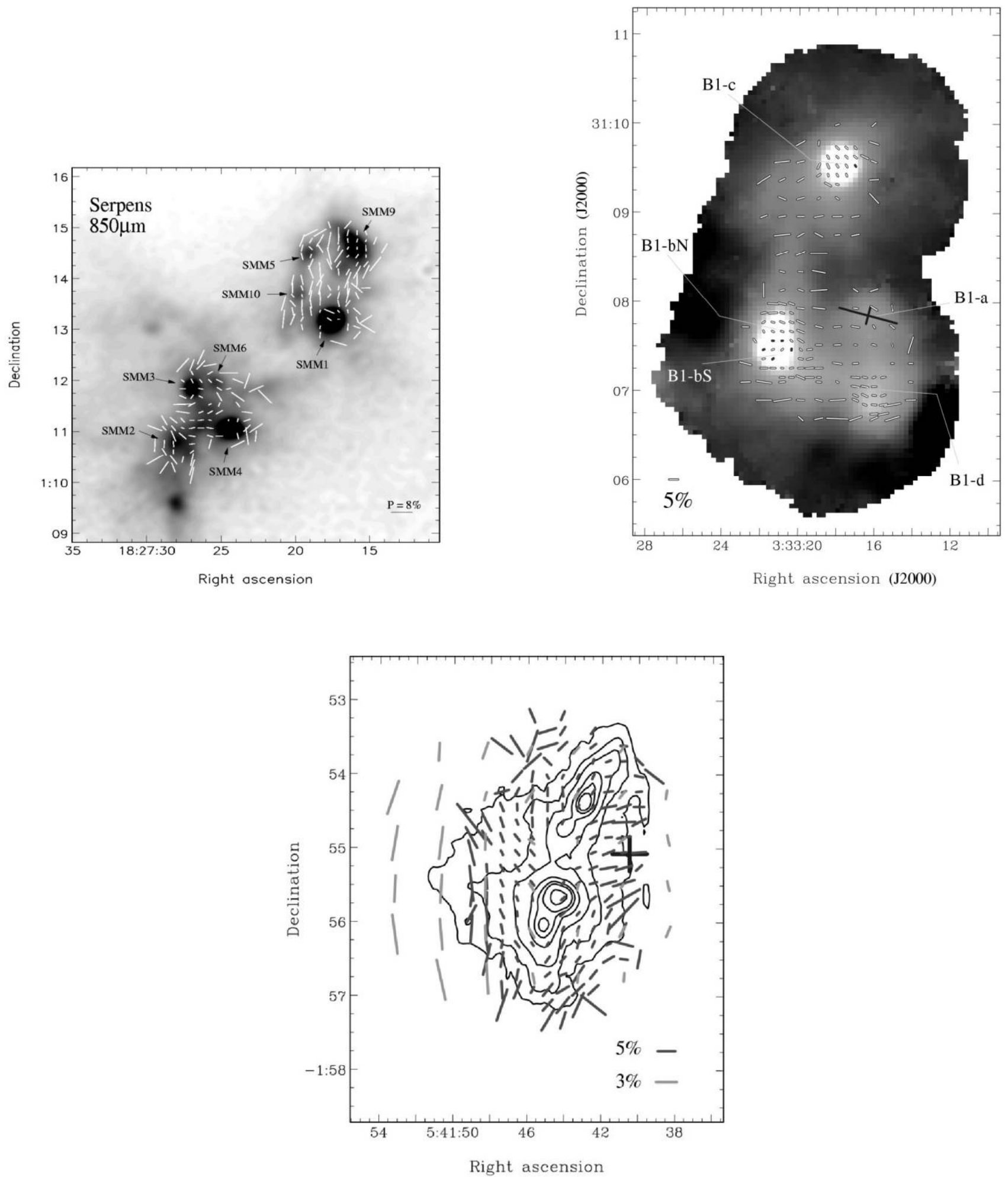

FIG. 16.-SCUBA polarization maps. These images show the importance of mapping the ambient environment around cores and the advantage of high resolution. Top left: SCUBA Serpens map (Davis et al. 2000) showing that the field structure can be more complex between the cores than their polarization patterns indicate. Top right: The same is true in Barnard 1 at center (Matthews \& Wilson 2002). Bottom: NGC 2024 shows that SCUBA observations (dark gray vectors) of extended structure in Orion (Matthews et al. 2002) reveal systematic variations missed in earlier lower resolution maps (light gray vectors; Dotson et al. 2000). This map reveals how critical resolution is to the effective mapping of magnetic field geometry. 
Models predict different relations between core morphology and polarization position angle, depending on field geometry (straight or helical) and core morphology (oblate, prolate, or triaxial). Since the outflow, core, and field orientations are all measured in two-dimensional projection, statistical corrections can be made for an ensemble of cores that are not applicable to individual objects.

\subsection{Models of Magnetic Field Geometry}

Polarized dust emission yields only the two-dimensional field geometry projected onto the plane of the sky. Using all three components from the survey (continuum, line, and dust polarimetry), we will generate a set of analytic models of the threedimensional field geometry of all 100 cores, as well as extended coherent structures such as filaments. This will be practical using a new generalized modeling code (Fiege 2005) that has already been applied to the case of a filamentary cloud (Fiege et al. 2004).

The most general question to be addressed is whether there are any quasi-static magnetic models of cores that can explain the polarization and continuum maps for a statistically significant number of sources, as some theoretical models predict (e.g., Mouschovias 1976; Tomisaka et al. 1988).

If all quasi-static models fail to provide adequate fits to the data, then the data could only be explained by MHD turbulence. Regardless of the outcome, the polarization data set will settle this important question in star formation theory. It will also provide a polarization catalog of legacy value that can be used as a definitive test for future models and simulations and serve as a powerful tool for their refinement.

The resulting modeling database will provide the theoretical counterpart to the observational data set, which would be augmented as new and improved models are developed. In principle, one could search for regions in parameter space where the allowed solution sets intersect for multiple cores. This would provide a way to search for preferred ranges of parameters, which would help to refine future models.

\subsection{Magnetic Field Strength}

The fractional polarization from dust yields no direct estimate of the magnetic field strength, since it is dependent on several additional unknowns (e.g., degree of grain alignment, grain shape, and composition). The field strength will be derived from the commonly used Chandrasekhar-Fermi (CF) method (Chandrasekhar \& Fermi 1953), using dispersion in polarization vectors (where high dispersion indicates a highly turbulent field and a weak mean field component), the line widths estimated from the HARP $\mathrm{C}^{18} \mathrm{O} /{ }^{13} \mathrm{CO}$ data, and the density from the SCUBA-2 fluxes (e.g., Crutcher et al. 2004; J. Kirk et al. 2006).

Simulations show that this estimate can be corrected for a statistical ensemble of objects to yield realistic estimates of the field strength (Ostriker et al. 2001; Heitsch et al. 2001). Due to measurements of line widths and dispersion estimates, we will be able to test effectively whether the cores with broader line widths show more dispersion, thereby determining the applicability of the CF method in molecular clouds.

\subsection{Large-Scale Fields and Turbulence}

The same extended regions will be mapped with POL-2 and HARP. The relation between the core field geometry and that of the larger scale structure in clustered and filamentary regions will be observed and modeled. Polarization maps can reveal abrupt changes in polarization direction (see Fig. 16), which indicate underlying changes in the magnetic field direction.

The gas dynamics from HARP $\mathrm{C}^{18} \mathrm{O} /{ }^{13} \mathrm{CO}$ observations will be used with the polarization maps to test predictions of magnetized simulations of turbulence (e.g., Heitsch et al. 2001; Ostriker et al. 2001; Padoan \& Nordlund 2002). Such largescale maps are the key to unraveling the overall geometry of the magnetic field.

\section{OTHER SURVEYS}

In parallel with this survey, there are a number of far-infrared surveys of the Gould Belt that are also being carried out. The two most relevant to this paper are those being carried out on the Spitzer Space Telescope and the Herschel Space Telescope.

The Spitzer Space Telescope (formerly SIRTF, the Space Infrared Telescope Facility; Werner et al. 2004) was launched on 2003 August 25. It consists of an $85 \mathrm{~cm}$ telescope and three cryogenically cooled science instruments, covering a wavelength range of 3-180 $\mu \mathrm{m}$. The three instruments are the Infrared Array Camera (IRAC; Fazio et al. 2004), the Infrared Spectrograph (IRS; Houck et al. 2004), and the Multiband Imaging Photometer for Spitzer (MIPS; Rieke et al. 2004). IRAC is a four-channel camera that provides simultaneous 5.12' $\times$ $5.12^{\prime}$ images at 3.6, 4.5, 5.8, and $8 \mu \mathrm{m}$. Each of the four detector arrays in the camera are $256 \times 256$ pixels in size. The IRS has four separate modules: a low-resolution, short-wavelength mode covering the 5.3-14 $\mu \mathrm{m}$ interval; a high-resolution, shortwavelength mode covering 10-19.5 $\mu \mathrm{m}$; a low-resolution, longwavelength mode for observations at $14-40 \mu \mathrm{m}$; and a highresolution, long-wavelength mode for $19-37 \mu \mathrm{m}$. MIPS consists of a $128 \times 128$ array for imaging at $24 \mu \mathrm{m}$, a $32 \times$ 32 array for imaging at $70 \mu \mathrm{m}$, and a $2 \times 20$ array for imaging at $160 \mu \mathrm{m}$.

One legacy program on Spitzer is currently mapping the same Gould Belt clouds as are described in this paper, using IRAC and MIPS. ${ }^{28}$ This is a follow-up to the "Cores to Disks" Spitzer Legacy Project (c2d; Evans et al. 2003; see also Allen et al. 2007; Guedel et al. 2007)

The Herschel Space Telescope (Pilbratt 2005a, 2005b) is a $3.5 \mathrm{~m}$ diameter, passively cooled telescope due to be launched in 2008. The science payload complement is comprised of the

\footnotetext{
${ }^{28}$ For more information, see http://www.cfa.harvard.edu/gouldbelt.
} 
Heterodyne Instrument for the Far-Infrared (HIFI; de Graauw et al. 2005), which is a very high resolution heterodyne spectrometer; the Photodetector Array Camera and Spectrometer (PACS; Poglitsch et al. 2005), an imaging photometer and medium-resolution grating spectrometer; and the Spectral and Photometric Imaging Receiver (SPIRE; Griffin et al. 2006), an imaging photometer and an imaging Fourier transform spectrometer.

One Guaranteed Time Program on Herschel will use PACS and SPIRE to map the same clouds in the Gould Belt at wavelengths of $75,170,250,350$, and $500 \mu \mathrm{m}$ (André \& Saraceno 2005). ${ }^{29}$

29 For a full description of this program, see http://starformationherschel.iap.fr.

\section{SUMMARY}

We have presented a program of observations that will be carried out by the JCMT using its new suite of instruments: SCUBA-2, HARP, and POL-2. These instruments will be used to survey the nearby star-forming regions in the Gould Belt to answer key questions in star formation research. The results of the survey will include legacy images and submillimeter source catalogs of the mapped regions. The data will be presented in a series of data papers, followed by a number of papers interpreting the data and addressing the science questions discussed above. The combination of this survey, together with those mentioned in the previous section, will provide a unique set of complementary data and a powerful legacy for many years to come.

\section{REFERENCES}

Allen, L., et al. 2007, in Protostars and Planets V, ed. B. Reipurth, D. Jewitt, \& K. Keil (Tucson: Univ. Arizona Press), 361

Alves, J. F., Lada, C. J., \& Lada, E. A. 2001, Nature, 409, 159

André, P., \& Saraceno, P. 2005, in the Dusty and Molecular Universe (ESA SP-577; Noordwijk: ESA), 179

André, P., Ward-Thompson, D., \& Barsony, M. 1993, ApJ, 406, 122 . 2000, in Protostars and Planets IV, ed. V. Mannings, S. S. Russell, \& A. Boss (Tucson: Univ. Arizona Press), 59

Ayliffe, B., Langdon, J. C., Cohl, H. S., \& Bate, M. R. 2007, MNRAS, 374,1198

Ballasteros-Paredes, J., Klessen, R. S., \& Vazquez-Semadeni, E. 2003, ApJ, 592, 188

Balsara, D., Ward-Thompson, D., \& Crutcher, R. M. 2001, MNRAS, 327,715

Bastien, P., Bissonnette, E., Ade, P. A. R., Pisano, G., Savini, G., Jenness, T., Johnstone, D., \& Matthews, B. 2005, JRASC, 99, 133

Bate, M. R., Bonnell, I. A., \& Bromm, V. 2003, MNRAS, 339, 577

Belloche, A., André, P., \& Motte, F. 2001, in ASP Conf. Ser. 243, From Darkness to Light: Origin and Evolution of Young Stellar Clusters, ed. T. Montmerle \& P. André (San Francisco: ASP), 313 Bensch, F., Stutzki, J., \& Ossenkopf, V. 2001, A\&A, 366, 636

Blaauw, A. 1991, in The Physics of Star Formation and Early Stellar Evolution, ed. C. J. Lada \& N. D. Kylafis (Dordrecht: Kluwer), 125

Bontemps, S., André, P., Terebey, S., \& Cabrit, S. 1996a, A\&A, 311, 858

Bontemps, S., Ward-Thompson, D., \& André, P. 1996b, A\&A, 314, 477

Brunt, C. M., Heyer, M. H., Vazquez-Semadeni, E., \& Pichardo, B. 2003, ApJ, 595, 824

Cambrésy, L. 1999, A\&A, 345, 965

Caselli, P., Walmsley, C. M., Tafalla, M., Dore, L., \& Myers, P. C. 1999, ApJ, 523, L165

Chandrasekhar, S., \& Fermi, E. 1953, ApJ, 118, 113

Clemens, D. P., \& Barvainis, R. 1988, ApJS, 68, 257

Clube, S. V. M. 1967, MNRAS, 137, 189

Comeron, F., \& Torra, J. 1992, A\&A, 261, 94 1994, A\&A, 281, 35

Comeron, F., Torra, J., \& Gomez, A. E. 1992, Ap\&SS, 187, 187

Crapsi, A., et al. 2005, A\&A, 439, 1023

Crutcher, R. M. 1999, ApJ, 520, 706

Crutcher, R. M., Nutter, D. J., Ward-Thompson, D., \& Kirk, J. M. 2004, ApJ, 600, 279
Dame, T. M., Hartmann, D., \& Thaddeus, P. 2001, ApJ, 547, 792

Davis, C. J., Chrysostomou, A., Matthews, H. E., Jenness, T., \& Ray, T. P. 2000, ApJ, 530, L115

de Graauw, T., et al. 2005, BAAS, 37, 1219

de Zeeuw, P. T., Hoogerwerf, R., de Bruijne, J. H. J., Brown, A. G. A., \& Blaauw, A. 1999, AJ, 117, 354

Di Francesco, J., Evans, N. J., II, Caselli, P., Myers, P. C., Shirley, Y., Aikawa, A., \& Tafalla, M. 2007, in Protostars and Planets V, ed. B. Reipurth, D. Jewitt, \& K. Keil (Tucson: Univ. Arizona), 17

Dobashi, K., Uehara, H., Kandori, R., Sakurai, T., Kaiden, M., Umemoto, T., \& Sato, F. 2005, PASJ, 57, S1

Dotson, J. L., Davidson, J., Dowell, C. D., Schleuning, D. A., \& Hildebrand, R. H. 2000, ApJS, 128, 335

Elmegreen, B. G. 2000, MNRAS, 311, L5

Evans, N. J., et al. 2003, PASP, 115, 965

Fazio, G., et al. 2004, ApJS, 154, 10

Fiege, J. D. 2005, in ASP Conf. Ser. 343, Astronomical Polarimetry: Current Status and Future Directions, ed. A. J. Adamson et al. (San Francisco: ASP), 171

Fiege, J. D., Johnstone, D., Redman, R. O., \& Feldman, P. A. 2004, ApJ, 616, 925

Frerking, M. A., Langer, W. D., \& Wilson, R. W. 1982, ApJ, 262, 590

Fuller, G. A., \& Ladd, E. F. 2002, ApJ, 573, 699

Goodman, A. A., Barranco, J. A., Wilner, D. J., \& Heyer, M. H. 1998a, Astrophys. Lett. Commun., 37, 109

- 1998b, ApJ, 504, 223

Goodwin, S. P., Whitworth, A. P., \& Ward-Thompson, D. 2004a, A\&A, 414, 633 2004b, A\&A, 423, 169

Gould, B. A. 1879, Uranometria Argentina, Resultados del Observatorio Nacional Argentino en Cordoba, Vol. 1 (Buenos Aires: P. E. Coni)

Greaves, J. S., Holland, W. S., \& Pound, M. W. 2003, MNRAS, 346, 441

Greaves, J. S., Holland, W. S., \& Ward-Thompson, D. 1997, ApJ, 480,255

Griffin, M. J., et al. 2006, Proc. SPIE, 6265, 7

Guedel, M., Padgett, D. L., \& Dougados, C. 2007, in Protostars and Planets V, ed. B. Reipurth, D. Jewitt, \& K. Keil (Tucson: Univ. Arizona Press), 329

Guillout, P., Sterzik, M. F., Schmitt, J. H. M. M., Motch, C., \& Neuhauser, R. 1998, A\&A, 337, 113 
Hartmann, L., Ballesteros-Paredes, J., \& Bergin, E. A. 2001, ApJ, 562,852

Hatchell, J., Richer, J. S., Fuller, D. A., Qualtrough, C. J., \& Ladd, E. F. 2007, A\&A, 468, 1009

Hatchell, J., Richer, J. S., Fuller, G. A., Qualtrough, C. J., Ladd, E. F., \& Chandler, C. J. 2005, A\&A, 440, 151

Heitsch, F., Zweibel, E. G., Mac Low, M.-M., Li, P., \& Norman, M. L. 2001, ApJ, 561, 800

Herschel, J. F. W. 1847, Results of Astronomical Observations Made at the Cape of Good Hope (London: Smith, Elder, and Co.)

Heyer, M. H., \& Schloerb, F. P. 1997, ApJ, 475, 173

Holland, W. S., et al. 1999, MNRAS, 303, 659 2006, Proc. SPIE, 6275, 45

Houck, J. R., et al. 2004, ApJS, 154, 18

Hovey, G. J., et al. 2000, Proc. SPIE, 4015, 114

Jappsen, A. K., \& Klessen, R. S. 2004, A\&A, 423, 1

Jessop, N. E., \& Ward-Thompson, D. 2001, MNRAS, 323, 1025

Jijina, J., Myers, P. C., \& Adams, F. C. 1999, ApJS, 125, 161

Johnstone, D., \& Bally, J. 1999, ApJ, 510, L49

Johnstone, D., Boonman, A. M. S., \& van Dishoeck, E. F. 2003, A\&A, 412, 157

Johnstone, D., Di Francesco, J., \& Kirk, H. 2004, ApJ, 611, L45

Johnstone, D., Fich, M., Mitchell, G., \& Moriarty-Schieven, G. 2001, ApJ, 559, 307

Johnstone, D., Matthews, H., \& Mitchell, G. F. 2006, ApJ, 639, 259

Johnstone, D., Wilson, C. D., Moriarty-Schieven, G., Joncas, G., Smith, G., Gregersen, E., \& Fich, M. 2000, ApJ, 545, 327

Jorgensen, J. K., Schoier, F. L., \& van Dishoeck, E. F. 2002, A\&A, 389,908

Kerton, C. R., Brunt, C. M., Jones, C. E., \& Basu, S. 2003, A\&A, 411, 149

Khalil, A., Joncas, G., \& Nekka, F. 2004, ApJ, 601, 352

Kirk, H., Johnstone, D., \& Di Francesco, J. 2006, ApJ, 646, 1009

Kirk, H., Johnstone, D., \& Tafalla, M. 2007, ApJ, in press

Kirk, J. M., Ward-Thompson, D., \& André, P. 2005, MNRAS, 360, 1506

Kirk, J. M., Ward-Thompson, D., \& Crutcher, R. M. 2006, MNRAS, 369,1445

Kroupa, P. 2001, MNRAS, 322, 231

Launhardt, R., Ward-Thompson, D., \& Henning, T. 1997, MNRAS, 288, L45

Lee, C. W., \& Myers, P. C. 1999, ApJS, 123, 233

Li, Z. Y., \& Nakamura, F. 2006, ApJ, 640, L187

Lynds, B. 1962, ApJS, 7, 1

Matthews, B. C., Fiege, J. D., \& Moriarty-Schieven, G. H. 2002, ApJ, 569,304

Matthews, B. C., \& Wilson, C. D. 2002, ApJ, 574, 822

Matthews, B. C., Wilson, C. D., \& Fiege, J. D. 2001, ApJ, 562, 400

Matthews, B. C., et al. 2007, PASP, 119, 842

Matzner, C. D. 2007, ApJ, 659, 1394

Matzner, C. D., \& Levin, Y. 2005, ApJ, 628, 817

Matzner, C. D., \& McKee, C. F. 2000, ApJ, 545, 364

McKee, C. F., \& Williams, J. P. 1997, ApJ, 476, 144

Moriarty-Schieven, G. H., Johnstone, D., Bally, J., \& Jenness, T. 2006, ApJ, 645, 357

Motte, F., André, P., Ward-Thompson, D., \& Bontemps, S. 2001, A\&A, 372, L41

Mouschovias, T. 1976, ApJ, 206, 753 . 1991, ApJ, 373, 169

Myers, P. C., \& Goodman, A. A. 1988, ApJ, 326, L27

Norman, C., \& Silk, J. 1980, ApJ, 238, 158
Nutter, D. J., \& Ward-Thompson, D. 2007, MNRAS, 374, 1413

Nutter, D. J., Ward-Thompson, D., \& André, P. 2005, MNRAS, 357, 975

- 2006, MNRAS, 368, 1833

Ossenkopf, V. 2002, A\&A, 391, 295

Ostriker, E. C., Stone, J. M., \& Gammie, C. F. 2001, ApJ, 546, 980

Padoan, P., \& Nordlund, A. 2002, ApJ, 576, 870

Pierce-Price, D., et al. 2000, ApJ, 545, L121

Pilbratt, G. 2005a, BAAS, 37, 1219

. 2005b, in the Dusty and Molecular Universe, ed. A. Wilson (ESA SP-577; Noordwijk: ESA), 3

Poglitsch, A., et al. 2005, BAAS, 207, 3502

Pöppel, W. G. L. 2001, in ASP Conf. Ser. 243, From Darkness to Light: Origin and Evolution of Young Stellar Clusters, ed. T. Montmerle \& P. André (San Francisco: ASP), 667

Redman, R. O., Feldman, P. A., Cote, S., Wyrowski, F., Carey, S. J., \& Egan, M. P. 2002, in ASP Conf. Ser. 267, Hot Star Workshop III: The Earliest Stages of Massive Star Birth, ed. P. A. Crowther (San Francisco: ASP), 409

Ridge, N., et al. 2006, AJ, 131, 2921

Rosolowsky, E. W., Goodman, A. A., Wilner, D. J., \& Williams, J. P. 1999, ApJ, 524, 887

Rieke, G., et al. 2004, ApJS, 154, 25

Salpeter, E. E. 1955, ApJ, 121, 161

Shu, F. H. 1977, ApJ, 214, 488

Silk, J. 1995, ApJ, 438, L41

Smith, H., et al. 2003, Proc. SPIE, 4855, 338

Stothers, R., \& Frogel, J. A. 1974, AJ, 79, 456

Stutzki, J., Bensch, F., Heithausen, A., Ossenkopf, V., \& Zielinsky, M. 1998, A\&A, 336, 697

Tomisaka, K., Ikeuchi, S., \& Nakamura, T. 1988, ApJ, 326, 208

van Dishoeck, E. F. 2006, Proc. Natl. Acad. Sci., 103, 12249

Vazquez-Semadeni, E., Kim, J., Shadmehri, M., \& Ballesteros-Paredes, J. 2005, ApJ, 618, 344

Visser, A. E., Richer, J. S., \& Chandler, C. J. 2002, AJ, 124, 2756

Walsh, A. J., Bourke, T. L., \& Myers, P. C. 2006, ApJ, 637, 860

Walsh, A. J., Myers, P. C., \& Burton, M. G. 2004, ApJ, 614, 194

Ward-Thompson, D. 2004, Observatory, 124, 362

Ward-Thompson, D., André, P., Crutcher, R., Johnstone, D., Onishi, T., \& Wilson, C. 2007, in Protostars and Planets V, ed. B. Reipurth, D. Jewitt, \& K. Keil (Tucson: Univ. Arizona Press), 33

Ward-Thompson, D., Chini, R., Krugel, E., André, P., \& Bontemps, S. 1995, MNRAS, 274, 1219

Ward-Thompson, D., Kirk, J. M., Crutcher, R. M., Greaves, J. S., Holland, W. S., \& André, P. 2000, ApJ, 537, L135

Ward-Thompson, D., Robson, E. I., Whittet, D. C. B., Gordon, M. A., Walther, D. M., \& Duncan, W. D. 1989, MNRAS, 241, 119

Ward-Thompson, D., Scott, P. F., Hills, R. E., \& André, P. 1994, MNRAS, 268, 276

Werner, M. W., et al. 2004, ApJS, 154, 1

Whitworth, A. P., Bate, M. R., Nordlund, A., Reipurth, B., \& Zinnecker, H. 2007, in Protostars and Planets V, ed. B. Reipurth, D. Jewitt, \& K. Keil (Tucson: Univ. Arizona Press), 459

Whitworth, A. P., \& Ward-Thompson, D. 2001, ApJ, 547, 317

Williams, J. P., de Geus, E. J., \& Blitz, L. 1994, ApJ, 428, 693

Wilson, B. A., Dame, T. M., Masheder, M. R. W., \& Thaddeus, P. 2005, A\&A, 430, 523

Wilson, T. L., \& Rood, R. 1994, ARA\&A, 32, 191

Wolf-Chase, G. A., Barsony, M., Wootten, H. A., Ward-Thompson, D., Lowrance, P. J., Kastner, J. H., \& McMullin, J. P. 1998, ApJ, 501, L193 